\title{
The Long-Run Behavior of Consumption and Wealth Dynamics in Complete Financial Market with Heterogeneous Investors
}

\author{
Darong Dai \\ Department of Economics, Texas A\&M University, College Station, TX 77843, USA \\ Correspondence should be addressed to Darong Dai; ddai@econmail.tamu.edu
}

Received 8 February 2014; Revised 19 June 2014; Accepted 24 June 2014; Published 14 July 2014

Academic Editor: Juan Manuel Peña

Copyright (C) 2014 Darong Dai. This is an open access article distributed under the Creative Commons Attribution License, which permits unrestricted use, distribution, and reproduction in any medium, provided the original work is properly cited.

\begin{abstract}
A type of complete financial market with finite and countable heterogeneous investors, that is, investors equipped with heterogeneous elasticities of intertemporal substitution, heterogeneous time discount rates, and also heterogeneous beliefs, is constructed and two main results are established. First, long-run behaviors, specifically golden rules or modified golden rules, about consumption path and wealth accumulation are investigated under uncertainty and in the sense of uniform topology. Second, inefficacy of temporary taxation policies, which are chosen to be consumption tax and wealth tax, is confirmed in the current financial market.
\end{abstract}

\section{Introduction}

Our goal in this paper is to explore the golden rule or modified golden rule properties of consumption and wealthaccumulation dynamics, as well as the effects of temporary taxation policies, which are chosen to be consumption tax and wealth tax, in a type of complete financial market with finite and countable heterogeneous investors (see, [1], e.g.), that is, investors with heterogeneous elasticities of intertemporal substitution (e.g., [2,3]), heterogeneous time discount rates (e.g., [2-4]), and heterogeneous beliefs (see, [2, 3, 5-8], and among others), choosing optimal consumption and portfolio strategy in an economy of infinite horizon. Golden rules about the consumption path, the wealth dynamics, and the combination of both are proved under uncertainty and in the sense of uniform topology, which would be regarded as the first innovation of the current paper. Furthermore, inefficacy of temporary taxation policies has also been confirmed in the current complete financial market, leading us to the second inspiration of the current paper.

In the past several decades, portfolio turnpikes (see [9-15], among others) in financial economics have been extensively studied and well-understood. Meanwhile, the concept of golden rule or modified golden rule (e.g., [16-21], among others) has been developed and plays a crucial role in studying optimal economic growth and optimal capital accumulation in macroeconomics. However, little attention up to the present has been paid to the golden rule or modified golden rule of consumption path and wealth accumulation in complete or incomplete financial market with heterogeneous investors. Noting that consumption strategy and wealth accumulation play the same, if not more, important role as that of portfolio choice in both capital asset pricing models (see [22-27], among others) and market selection theory (e.g., $[2,7,28-35]$, among others), the current paper is encouraged to meet the gap and investigate the long-run behavior of consumption and wealth dynamics in a type of complete financial market with heterogeneous investors.

Indeed, the current paper confirms the following strong conclusion: both optimal consumption path and optimal wealth dynamics are long-run golden rules in the sense of uniform topology and in the corresponding nonstationary environment, regardless of the fact that there are many heterogeneous investors in the economy. In other words, the uniform topology golden rules demonstrated in the present paper are robust to the types of investors in the market as long as they all exhibit the same type of CRRA preferences. Nonetheless, these golden rules are not turnpikes because they are sensitive to initial conditions of the corresponding dynamics [36-38]. And hence, naturally, an open question comes up: when these golden rules are also turnpikes? The exploration of this question will be left to future study. 
The second goal of this paper is to study the effect of taxation policies, which are specifically chosen to be consumption tax and wealth tax, to optimal consumption strategy. As in the literatures of Yano [38, 39] and Kondo [40], the current paper proves the conclusion of inefficacy of temporary taxation policies in a type of complete financial market with heterogeneous investors in comparatively weak conditions, which are different from those of Yano [38, 39] and Kondo [40] due to the dynamic competitive-equilibrium framework they employed.

In addition, although both this paper and Jin [13] investigate the long-run behavior of consumption process in a continuous-time finance model, it is worthwhile mentioning that our results are essentially different from those of Jin [13] in the following aspects. First, Jin mainly proves the portfolio turnpike theorems in a continuous-time model, which however is not our focus in this paper. In particular, Jin proves related turnpike properties, whereas our paper confirms the relevant golden rule properties, and we already know that golden run property is generally weaker than turnpike property. Second, Jin shows the related convergence between processes under different utility-function assumptions, that is, general utility functions, such that the inverse functions of the derivative of utility functions for consumption and investment belong to a special subclass of regularly varying functions and power utility functions, whereas we show the convergence between processes under arbitrary decisions and optimal decisions. Third, Jin just confirms the convergence of final wealth process while the present paper shows convergence for the entire path of wealth accumulation. And fourth, we show a much stronger convergence in the sense of uniform topology while this desired property generally cannot be satisfied in the paper of Jin.

Finally, we would like to indicate the differences and also similarity between our investigation and the well-known papers of Sandroni [30] and Blume and Easley [7]. First, the fundamental issue investigated in these papers is distinct with our paper, that is, they all focus on the market-selection theory in complete or incomplete markets, while the current study purely characterizes the long-run behaviors of wealth and consumption processes of heterogeneous investors. Second, it is easy to see that the current model environment is different from the above papers; in particular, they use discretetime models and we use a continuous-time model driven by Brownian motions, and it is easy to see that our model intrinsically leads us to more complicated computations in demonstrating the corresponding convergence result. Third, although these papers emphasize the proof of corresponding convergence, their convergence results are just in point-wise sense and hence are much weaker than the present meansquare convergence in uniform topology sense. Last but not least, even though the huge differences exist in modeling environment and focus, our result induces similar intuitive implications as these papers, that is, wealth-accumulation process will uniformly converge to the equilibrium path under optimal decision and hence market selection will prefer those make accurate predications in complete markets.

The rest of the paper is organized as follows. Section 2 presents the basic definitions and assumptions about the complete financial market facing us; Section 3 solves the individual optimization problem facing heterogeneous investors, that is, both optimal consumption-portfolio strategy and optimal wealth dynamics are derived; Section 4 proves the uniform topology golden rules in the current financial market based upon the results in Section 3. There is a brief concluding section. All proofs appear in the Appendix.

\section{Definitions and Assumptions}

Suppose that there are $I$ heterogeneous agents in the economy. Perfect foresight assumption is employed and we denote by $\left(\Omega^{i}, \mathscr{F}^{i},\left\{\mathscr{F}_{t}^{i}\right\}_{t \geq 0}, \mathbb{P}^{i}\right)$ the complete and filtered probability space with $\mathbb{F}^{i}=\left\{\mathscr{F}_{t}^{i}\right\}_{t \geq 0}$ denoting the filtration and the sigmaalgebra $\mathscr{F}_{t}^{i}$ generated by a $d$-dimensional standard Brownian motion $\left(W^{i}(s), 0 \leq s \leq t\right)$. Here, and throughout the current paper, $\mathbb{E}^{i}$ is used to denote the expectation operator with respect to the probability law $\mathbb{P}^{i}$ for $\forall i=1,2, \ldots, I$. Moreover, we are provided with another stochastic basis $\left(\Omega, \mathscr{F},\left\{\mathscr{F}_{t}\right\}_{t \geq 0}, \mathbb{P}\right)$, where $\Omega=\prod_{i=1}^{I} \Omega^{i}, \mathscr{F}=\otimes_{i=1}^{I} \mathscr{F}^{i}, \mathscr{F}_{t}=$ $\otimes_{i=1}^{I} \mathscr{F}_{t}^{i}, \mathbb{P}=\otimes_{i=1}^{I} \mathbb{P}^{i}$ and we let $\mathbb{F}=\left\{\mathscr{F}_{t}\right\}_{t \geq 0}$ denote the corresponding filtration satisfying the usual conditions. We further denote by $\mathbb{E}$ the expectation operator with respect to the probability law $\mathbb{P}$.

We define the canonical Lebesgue measure $\mu$ on measure space $\left(\mathbb{R}_{+}, \mathfrak{B}\left(\mathbb{R}_{+}\right)\right)$with $\mathbb{R}_{+}=[0, \infty), \mathbb{R}_{++}=(0, \infty)$ and $\mathfrak{B}\left(\mathbb{R}_{+}\right)$the Borel sigma-algebra, and also the corresponding regular properties about Lebesgue measure are supposed to be fulfilled. Thus, we can define the following product measure spaces $\left(\Omega^{i} \times \mathbb{R}_{+}, \mathbb{F}^{i} \otimes \mathfrak{B}\left(\mathbb{R}_{+}\right)\right)$and $\left(\Omega \times \mathbb{R}_{+}, \mathbb{F} \otimes\right.$ $\mathfrak{B}\left(\mathbb{R}_{+}\right)$) with corresponding product measures being $d \mu \otimes d \mathbb{P}^{i}$ and $d \mu \otimes d \mathbb{P}$, respectively, for $\forall i=1,2, \ldots, I$.

Now, based upon the probability space $\left(\Omega^{i}, \mathscr{F}^{i}, \mathbb{F}^{i}, \mathbb{P}^{i}\right)$, we define the complete financial market as follows:

$$
\begin{gathered}
d B(t)=r(t) B(t) d t, \quad B(0)=1 . \\
d S_{j}^{i}(t)=S_{j}^{i}(t)\left[b_{j}^{i}(t) d t+\sum_{k=1}^{d} \sigma_{j k}(t) d W_{k}^{i}(t)\right], \quad S_{j}^{i}(0)>0,
\end{gathered}
$$

where $B(t)$ denotes the price process of a safe or riskless investment, for example, bank account, and $S_{j}^{i}(t)$ denotes the price process of a risky investment, for instance, the stock, for $j=1,2, \ldots, m$ and $\forall i=1,2, \ldots, I$. And $r(t), b_{j}^{i}(t)$, $\sigma_{j k}(t) \in \mathbb{R}$ denote the riskless interest rate, the expectation return rate of the stock and the market volatility in period $t$, respectively, for $\forall i=1,2, \ldots, I, j=1,2, \ldots, m$ and $k=$ $1,2, \ldots, d$. In particular, if we let $b_{j}(t)$ represent the true value of market mean return of stock $j$, then we get $\mathbb{E}^{i}\left[b_{j}(t) \mid \mathscr{F}_{t}^{i}\right]=$ $b_{j}^{i}(t)>($ res. $=$ or $<) b_{j}(t)$ if individual $i$ is an optimistic (res. rational or pessimistic) investor, which reflects heterogeneous beliefs in the current financial market. Moreover, all of the above processes are supposed to be $\mathbb{F}^{i} \otimes \mathfrak{B}\left(\mathbb{R}_{+}\right)$-progressively 
measurable. Then, we can have the following SDE of wealth accumulation:

$$
\begin{aligned}
d X^{i}(t)= & \left(1-\tau_{X}(t)\right) X^{i}(t) \\
\times & {\left[\sum_{j=1}^{m} \pi_{j}^{i}(t) \frac{d S_{j}^{i}(t)}{S_{j}^{i}(t)}+\left(1-\sum_{j=1}^{m} \pi_{j}^{i}(t)\right) r(t) d t\right] } \\
& -\left(1+\tau_{c}(t)\right) c^{i}(t) d t \\
= & \left\{\left(1-\tau_{X}(t)\right) X^{i}(t)\right. \\
& \times\left[\pi^{i}(t)^{\top}\left(b^{i}(t)-r(t) \mathbf{1}\right)+r(t)\right] \\
& \left.-\left(1+\tau_{c}(t)\right) c^{i}(t)\right\} d t \\
& +\left(1-\tau_{X}(t)\right) X^{i}(t) \pi^{i}(t)^{\top} \sigma(t) d W^{i}(t) \\
= & f^{i}\left(X^{i}(t)\right) d t+g^{i}\left(X^{i}(t)\right) d W^{i}(t),
\end{aligned}
$$

subject to $X^{i}(0)=x^{i}>0, W^{i}(0)=\mathbf{1}_{0}=(0,0, \ldots, 0)^{\top} \mathbb{P}^{i}$ a.s., and $\tau_{X}, \tau_{c}$ denote the wealth and consumption tax rates, respectively. Let $\pi^{i}(t)=\left(\pi_{1}^{i}(t), \ldots, \pi_{m}^{i}(t)\right)^{\top}$ and let $c^{i}(t)$ represent portfolio strategy and consumption process, respectively. And as usual, $b^{i}(t)=\left(b_{1}^{i}(t), \ldots, b_{m}^{i}(t)\right)^{\top}, \mathbf{1}=$ $(1,1, \ldots, 1)^{\top}, W^{i}(t)=\left(W_{1}^{i}(t), \ldots, W_{d}^{i}(t)\right)^{\top}$, where the superscript " " denotes transpose, and $\sigma(t)=\left(\sigma_{j k}(t)\right) \in \mathbb{R}^{m \times d}$ denotes a bounded matrix. Furthermore, $X^{i}(t)$ is assumed to be $\mathbb{F}^{i} \otimes \mathfrak{B}\left(\mathbb{R}_{+}\right)$-adapted and all the remaining processes are $\mathbb{F}^{i} \otimes \mathfrak{B}\left(\mathbb{R}_{+}\right)$-progressively measurable for $\forall i=1,2, \ldots, I$. If denoted in matrix form, we can get

$$
d X(t)=f(X(t)) d t+g(X(t)) d W(t),
$$

in which $X(t)$ is assumed to be $\mathbb{F} \otimes \mathfrak{B}\left(\mathbb{R}_{+}\right)$-adapted and both $f(X(t))$ and $g(X(t))$ are supposed to be $\mathbb{F} \otimes \mathfrak{B}\left(\mathbb{R}_{+}\right)$progressively measurable. We employ the following assumptions in the model.

Assumption 1. The initial conditions $X^{i}(0)=x^{i}>0(\forall i=$ $1,2, \ldots, I)$ are supposed to be deterministic and bounded.

Assumption 2. The following linear growth and local Lipschitz continuity conditions are satisfied, respectively,

$$
\begin{gathered}
\left|f^{i}\left(y^{i}\right)\right|^{2}+\left\|g^{i}\left(y^{i}\right)\right\|_{2}^{2} \leq C^{i}\left(1+\left|y^{i}\right|^{2}\right), \\
\|f(y)\|_{2}^{2}+\|g(y)\|_{2}^{2} \leq C\left(1+\|y\|_{2}^{2}\right),
\end{gathered}
$$

for some constants $C^{i}, C<\infty$. And

$$
\begin{gathered}
\left|f^{i}\left(y^{i}\right)-f^{i}\left(z^{i}\right)\right|^{2} \vee\left\|g^{i}\left(y^{i}\right)-g^{i}\left(z^{i}\right)\right\|_{2}^{2} \leq L_{R^{i}}^{i}\left|y^{i}-z^{i}\right|^{2}, \\
\|f(y)-f(z)\|_{2}^{2} \vee\|g(y)-g(z)\|_{2}^{2} \leq L_{R}\|y-z\|_{2}^{2},
\end{gathered}
$$

for given constants $R^{i}, R>0,\left|y^{i}\right| \vee\left|z^{i}\right| \leq R^{i},\|y\|_{2} \vee\|z\|_{2} \leq$ $R$, and constants $L_{R^{i}}^{i}, L_{R}<\infty$ depend only on $R^{i}$ and $R$, respectively, for all $y^{i}, z^{i} \in \mathbb{R}_{++}, y, z \in \mathbb{R}_{++}^{I}$, for $\forall i=$ $1,2, \ldots, I$.

Remark 3. (i) Provided Assumption 2, the existence and uniqueness of strong solutions of the SDEs in (3) and (4) are ensured.

(ii) Assumption 2 is indeed weak in the following sense, that is, conditions (7) can be naturally satisfied for any $C^{1}$ functions due to the mean value theorem.

(iii) Here, and throughout the current paper, $|\cdot|$ is used to represent absolute value, $\|\cdot\|_{2}$ is used to denote both the Euclidean vector norm and the Frobenius (or trace) matrix norm, and $\langle\cdot, \cdot\rangle$ is used to denote the scalar product.

Assumption 4. The real symmetric matrix $\sigma(t) \sigma(t)^{\top}$ is assumed to be bounded and invertible throughout the current paper.

Now, as a preparation for solving individual optimization problem defined in the following section, we, as usual, provide the following formal definition,

Definition 5 (Markov admissible strategy). We call the control variable $\left(\pi^{i}(t), c^{i}(t)\right) \in[0,1]^{m} \times \mathbb{R}_{+}$an admissible strategy if the corresponding wealth process $X^{i}(t, \omega) \geq 0 d \mu \otimes d \mathbb{P}^{i}$ a.e. and we further call it a Markov admissible strategy if it satisfies Markov property of memorylessness, and then we define the set of Markov admissible strategy as $\mathscr{A}^{i}$ for $\forall i=$ $1,2, \ldots, I$.

Here, and throughout the current paper, we just consider Markov admissible strategies for the investors in the present complete financial market. In particular, we will derive in the following section the corresponding Markov admissible strategies for these investors by employing stochastic dynamic programming.

\section{Individual Optimization Problem}

We, as usual and without loss of any generality, suppose that the agents exhibit constant relative risk aversion (CRRA) preferences, and the individual optimization problem reads as follows:

$$
\max _{\left(\pi^{i}(t), c^{i}(t)\right) \in \mathscr{A ^ { i }}} \mathbb{E}_{\left(s, x^{i}\right)}^{i}\left[\int_{s}^{\infty} e^{-\rho^{i}(t-s)} \frac{c^{i}(t)^{\gamma^{i}}}{\gamma^{i}} d t\right]
$$

s.t.

$$
\begin{aligned}
d X^{i}(t)=\{ & \left(1-\tau_{X}(t)\right) X^{i}(t) \\
& \times\left[\pi^{i}(t)^{\top}\left(b^{i}(t)-r(t) \mathbf{1}\right)+r(t)\right] \\
& \left.-\left(1+\tau_{c}(t)\right) c^{i}(t)\right\} d t \\
+ & \left(1-\tau_{X}(t)\right) X^{i}(t) \pi^{i}(t)^{\top} \sigma(t) d W^{i}(t), \\
& X^{i}(0)=x^{i}>0, \quad \mathbb{P}^{i} \text {-a.s., }
\end{aligned}
$$


where $\mathbb{E}_{\left(s, x^{i}\right)}^{i}$ is defined in the precious section with initial conditions $\left(s, x^{i}\right) \in \mathbb{R}_{+} \times \mathbb{R}_{++}, \rho^{i}>0$ denotes the subjective discount factor. and $\gamma^{i} \in(0,1)$ is a constant with $1-\gamma^{i}$ denoting relative risk aversion coefficient for $\forall i=1,2, \ldots, I$.

Employing the classical technique of dynamic programming, the individual optimization problem defined in (8) is explicitly solved and the following proposition is, thus, established.

Proposition 6. Provided Assumption 4, then the optimal portfolio reads as follows:

$$
\widehat{\pi}^{i}(t)=\frac{1}{\left(1-\tau_{X}(t)\right)\left(1-\gamma^{i}\right)}\left(\sigma(t) \sigma(t)^{\top}\right)^{-1}\left(b^{i}(t)-r(t) \mathbf{1}\right),
$$

and if

$$
\begin{aligned}
\rho^{i}> & \left(1-\tau_{X}(t)\right)\left[\hat{\pi}^{i}(t)^{\top}\left(b^{i}(t)-r(t) \mathbf{1}\right)+r(t)\right] \gamma^{i} \\
& +\frac{1}{2}\left(1-\tau_{X}(t)\right)^{2} \hat{\pi}^{i}(t)^{\top} \sigma(t) \sigma(t)^{\top} \hat{\pi}^{i}(t) \gamma^{i}\left(\gamma^{i}-1\right),
\end{aligned}
$$

then the optimal consumption strategy is given in the feedback form, that is,

$$
\widehat{c}^{i}(t)=\left[\left(1+\tau_{c}(t)\right) \gamma^{i} C^{i}(t)\right]^{1 /\left(\gamma^{i}-1\right)} \widehat{X}^{i}(t),
$$

where

$$
\begin{aligned}
& C^{i}(t)= \frac{\left(1-\gamma^{i}\right)^{1-\gamma^{i}}}{\gamma^{i}\left(1+\tau_{c}(t)\right)^{\gamma^{i}}} \\
& \times\left\{\rho^{i}-\left(1-\tau_{X}(t)\right)\right. \\
& \quad \times\left[\hat{\pi}^{i}(t)^{\top}\left(b^{i}(t)-r(t) \mathbf{1}\right)+r(t)\right] \gamma^{i} \\
& \quad+\frac{1}{2}\left(1-\tau_{X}(t)\right)^{2} \hat{\pi}^{i}(t)^{\top} \sigma(t) \\
&\left.\quad \times \sigma(t)^{\top} \hat{\pi}^{i}(t) \gamma^{i}\left(1-\gamma^{i}\right)\right\}^{\gamma^{i}-1},
\end{aligned}
$$

and $\widehat{X}^{i}(t)$ is a strong solution of SDE

$$
\begin{aligned}
d \widehat{X}^{i}(t)= & \widehat{X}^{i}(t) \\
& \times\left\{\left(1-\tau_{X}(t)\right)\left[\hat{\pi}^{i}(t)^{\top}\left(b^{i}(t)-r(t) \mathbf{1}\right)+r(t)\right]\right. \\
& \left.\quad-\left(1+\tau_{c}(t)\right)\left[\left(1+\tau_{c}(t)\right) \gamma^{i} C^{i}(t)\right]^{1 /\left(\gamma^{i}-1\right)}\right\} d t \\
& +\left(1-\tau_{X}(t)\right) \widehat{X}^{i}(t) \hat{\pi}^{i}(t)^{\top} \sigma(t) d W^{i}(t) \\
= & \widehat{f}^{i}\left(\widehat{X}^{i}(t)\right) d t+\hat{g}^{i}\left(\widehat{X}^{i}(t)\right) d W^{i}(t),
\end{aligned}
$$

subject to $\widehat{X}^{i}(0)=x^{i}>0 \mathbb{P}^{i}$-a.s. for $\forall i=1,2, \ldots, I$.

Proof. See Appendix A.

\section{Uniform Topology Golden Rules}

By Proposition 6, we get the optimal paths of wealth accumulation as follows:

$$
\begin{aligned}
d \widehat{X}^{i}(t) & =\widehat{f}^{i}\left(\widehat{X}^{i}(t)\right) d t+\widehat{g}^{i}\left(\widehat{X}^{i}(t)\right) d W^{i}(t), \\
\widehat{X}^{i}(0) & =\widehat{x}^{i}>0, \quad \mathbb{P}^{i} \text {-a.s., } \forall i=1,2, \ldots, I,
\end{aligned}
$$

which can be rewritten in the following matrix form:

$$
\begin{gathered}
d \widehat{X}^{i}(t)=\widehat{f}\left(\widehat{X}^{i}(t)\right) d t+\hat{g}\left(\widehat{X}^{i}(t)\right) d W(t) \\
\widehat{X}^{i}(0)=\hat{x}>0, \quad \mathbb{P} \text {-a.s. }
\end{gathered}
$$
tions.

To prove the golden rules, we need the following assumpAssumption 7. The initial conditions $\widehat{X}^{i}(0)=\widehat{x}^{i}>0(\forall i=$ $1,2, \ldots, I)$ are supposed to be deterministic and bounded.

Assumption 8. There exist constants $L_{\hat{f}}, L_{\widehat{g}}>0$ such that

$$
\begin{gathered}
\langle\hat{y}-\widehat{z}, \widehat{f}(\widehat{y})-\widehat{f}(\widehat{z})\rangle \leq L_{\hat{f}}\|\hat{y}-\widehat{z}\|_{2}^{2}, \\
\|\widehat{g}(\widehat{y})-\widehat{g}(\widehat{z})\|_{2}^{2} \leq L_{\hat{g}}\|\hat{y}-\widehat{z}\|_{2}^{2},
\end{gathered}
$$

for $\forall \hat{y}, \widehat{z} \in \mathbb{R}_{+}^{I}$.

Remark 9. The inequality in (16) is the well-known one-sided Lipschitz condition.

It follows from Assumption 8 that

$$
\begin{aligned}
\langle\hat{f}(\hat{y}), \hat{y}\rangle & =\langle\hat{f}(\hat{y})-\hat{f}(0), \hat{y}\rangle+\langle\hat{f}(0), \hat{y}\rangle \leq L_{\hat{f}}\|\hat{y}\|_{2}^{2}, \\
\|\hat{g}(\hat{y})\|_{2}^{2} & \leq 2\|\hat{g}(0)\|_{2}^{2}+2\|\hat{g}(\hat{y})-\hat{g}(0)\|_{2}^{2} \leq 2 L_{\hat{g}}\|\hat{y}\|_{2}^{2} .
\end{aligned}
$$

Thus, we directly give the following assumption.

Assumption $5^{\prime}$. There exists a constant $\widehat{L}=L_{\widehat{f}} \vee L_{\widehat{g}}$ such that

$$
\langle\widehat{f}(\widehat{y}), \hat{y}\rangle \vee\|\widehat{g}(\hat{y})\|_{2}^{2} \leq \widehat{L}\|\hat{y}\|_{2}^{2},
$$

for $\forall \hat{y} \in \mathbb{R}_{+}^{I}$.

Given the above assumptions, the following lemma is derived.

Lemma 10. Given the optimal wealth dynamics defined in (15) and based upon Assumptions 7 and $5^{\prime}$, then for $\forall p \in \mathbb{N}, p \geq 2$ and for any given $T \geq 0$, there is a constant $\widehat{e}=\widehat{e}(\widehat{x}, p, T)>0$ such that

$$
\mathbb{E}\left[\sup _{0 \leq t \leq T}\left\|\widehat{X}^{i}(t)\right\|_{2}^{p}\right] \leq \widehat{e}
$$

Proof. See Appendix B. 
Moreover, we give the following assumption.

Assumption 11. There exist constants $\widehat{L}^{i}, L^{i}$, and $L>0$ such that

$$
\begin{gathered}
\left\langle\widehat{f}^{i}\left(\widehat{y}^{i}\right), \widehat{y}^{i}\right\rangle \vee\left\|\widehat{g}^{i}\left(\widehat{y}^{i}\right)\right\|_{2}^{2} \leq \widehat{L}^{i}\left|\hat{y}^{i}\right|^{2}, \\
\left\langle f^{i}\left(y^{i}\right), y^{i}\right\rangle \vee\left\|g^{i}\left(y^{i}\right)\right\|_{2}^{2} \leq L^{i}\left|y^{i}\right|^{2}, \\
\langle f(y), y\rangle \vee\|g(y)\|_{2}^{2} \leq L\|y\|_{2}^{2},
\end{gathered}
$$

for $\forall y^{i}, \widehat{y}^{i} \in \mathbb{R}_{+}(\forall i=1,2, \ldots, I)$ and $\forall y \in \mathbb{R}_{+}^{I}$.

Thus, similar to the proof of Lemma 10, we get the following lemma,

Lemma 12. Given the original wealth dynamics and the optimal wealth dynamics defined in (3), (4), and (13'), respectively. Based upon Assumptions 1, 7, and 11, and for $\forall p \in \mathbb{N}, p \geq 2$ and for any given $T \geq 0$, there are constants $e=e(x, p, T)$, $e^{i}=e^{i}\left(x^{i}, p, T\right)$, and $\hat{e}^{i}=\hat{e}^{i}\left(\hat{x}^{i}, p, T\right)>0$ such that

$$
\begin{gathered}
\mathbb{E}\left[\sup _{0 \leq t \leq T}\|X(t)\|_{2}^{p}\right] \leq e, \quad \mathbb{E}^{i}\left[\sup _{0 \leq t \leq T}\left|X^{i}(t)\right|^{p}\right] \leq e^{i}, \\
\mathbb{E}^{i}\left[\sup _{0 \leq t \leq T}\left|\widehat{X}^{i}(t)\right|^{p}\right] \leq \hat{e}^{i},
\end{gathered}
$$

for $\forall i=1,2, \ldots, I$.

Noting that $\hat{f}^{i}, \hat{g}^{i}, \widehat{f}$, and $\widehat{g}$ are all $C^{1}$ functions, thus, by the mean value theorem, we get the following local Lipschitz continuity property,

Condition 1 (local Lipschitz continuity). For any given constants $\widehat{R}^{i}, \widehat{R}>0$, there exist constants $L_{\widehat{R}^{i}}^{i}, L_{\widehat{R}}>0$ such that

$$
\begin{gathered}
\left|\widehat{f}^{i}\left(\widehat{y}^{i}\right)-\widehat{f}^{i}\left(\widehat{z}^{i}\right)\right|^{2} \vee\left\|\widehat{g}^{i}\left(\hat{y}^{i}\right)-\widehat{g}^{i}\left(\widehat{z}^{i}\right)\right\|_{2}^{2} \leq L_{\widehat{R}^{i}}^{i}\left|\hat{y}^{i}-\widehat{z}^{i}\right|^{2}, \\
\|\hat{f}(\widehat{y})-\widehat{f}(\widehat{z})\|_{2}^{2} \vee\|\hat{g}(\widehat{y})-\hat{g}(\widehat{z})\|_{2}^{2} \leq L_{\widehat{R}}\|\widehat{y}-\widehat{z}\|_{2}^{2},
\end{gathered}
$$

for $\left|\widehat{y}^{i}\right| \vee\left|\widehat{z}^{i}\right| \leq \widehat{R}^{i},\|\widehat{y}\|_{2} \vee\|\widehat{z}\|_{2} \leq \widehat{R}$, and for all $\widehat{y}^{i}, \widehat{z}^{i} \in$ $\mathbb{R}_{++}(\forall i=1,2, \ldots, I), \widehat{y}, \widehat{z} \in \mathbb{R}_{++}^{I}$.

And for the sake of simplicity, we need the following assumption

Assumption 13. There exist constants $K_{i}, K>0$ such that

$$
\begin{gathered}
\left|\widehat{f}^{i}\left(\hat{y}^{i}\right)-f^{i}\left(\hat{y}^{i}\right)\right|^{2} \vee\left\|\hat{g}^{i}\left(\hat{y}^{i}\right)-g^{i}\left(\hat{y}^{i}\right)\right\|_{2}^{2} \leq K_{i}\left|\hat{y}^{i}\right|^{2}, \\
\|\hat{f}(\hat{y})-f(\hat{y})\|_{2}^{2} \vee\|\hat{g}(\hat{y})-g(\hat{y})\|_{2}^{2} \leq K\|\hat{y}\|_{2}^{2},
\end{gathered}
$$

for $\forall \widehat{y}^{i} \in \mathbb{R}_{++}, \forall \hat{y} \in \mathbb{R}_{++}^{I}$ and for $\forall i=1,2, \ldots, I$.

Now, based on Lemmas 10 and 12, the following uniform topology golden rule is established.
Theorem 14 (uniform topology golden rule). Provided Assumptions 1, 2, 7, 5', and 13, and Condition 1, then for $\forall \bar{M}=R \vee \widehat{R}$, where $R$ and $\widehat{R}$ appear in Assumption 2 and Condition 1, respectively, for any given $T \geq 0$, and $\forall \varepsilon>0$, there exist $\delta_{1}(T, \bar{M}), \delta_{2}(T, \bar{M})>0$ (for any given $T$ and $\bar{M}$ ), if $\vee_{i=1}^{I} \hat{x}^{i}<\delta_{1}(T, \bar{M})$ and $\|\widehat{x}-x\|_{2}^{2}<\delta_{2}(T, \bar{M})$, one must have

$$
\mathbb{E}\left[\sup _{0 \leq t \leq T}\|\widehat{X}(t)-X(t)\|_{2}^{2}\right] \leq \varepsilon .
$$

Moreover, if we let $\lim _{T \rightarrow \infty} \delta_{i}(T, \bar{M})=0$ (for any given $\bar{M}, \forall i=$ $1,2)$, then one has

$$
\mathbb{E}\left[\lim _{T \rightarrow \infty} \sup _{0 \leq t \leq T}\|\widehat{X}(t)-X(t)\|_{2}^{2}\right]=0 .
$$

Therefore, mean-square convergence in uniform topology is confirmed for the current wealth accumulation paths.

Proof. See Appendix C.

Remark 15. This theorem is about the asymptotic properties of two wealth processes, $X^{i}(t)$ and $\widehat{X}^{i}(t)$, in which $\widehat{X}^{i}(t)$ is the strong solution to the SDE (14) that is evaluated at the optimal portfolio and consumption strategies and $X^{i}(t)$ is the strong solution of the SDE (2) but since those conditions are introduced before the utility function as well as the optimal decisions, it implies that the implicit portfolio and consumption processes are arbitrary. Therefore, Theorem 14 demonstrates that an arbitrary wealth process will uniformly converge to the optimal wealth-accumulation process in mean-square sense as long as the initial level of wealth is strictly controlled. Also, as the well-known argument of Yano [38] shows that Theorem 14 cannot be regarded as a uniform topology turnpike theorem, it, nevertheless, can be interpreted as a stability theorem. Actually, Theorem 14 proves both Liapounov stability (see, $[38,41]$ ) or dual Liapounov stability (e.g., [38, 39]) and asymptotic stability (e.g., [38, 42 ], and among others) of the optimal wealth dynamics under uncertainty and in the sense of uniform topology. In particular, golden rule is a weaker concept relative to the turnpike, that is, the former is allowed to depend on initial conditions while the latter does not in the process of convergence. Nevertheless, both golden rules and turnpikes refer to equilibrium paths evaluated at optimal strategies of individuals, that is, they represent desired paths.

Moreover, based upon Lemma 12 and similar to the proof of Theorem 14, the following theorem is derived.

Theorem 16 (uniform topology golden rule). Provided Assumptions 1, 2, 7, and 13, and Condition 1, then for $\forall \bar{M}^{i}=$ $R^{i} \vee \widehat{R}^{i}$, where $R^{i}$ and $\widehat{R}^{i}$ appear in Assumption 2 and Condition 1, respectively, and for any given $T \geq 0$, and $\forall \varepsilon^{i}>0$, there exist $\delta_{1}^{i}\left(T, \bar{M}^{i}\right), \delta_{2}^{i}\left(T, \bar{M}^{i}\right)>0$ (for any given $T$ and $\bar{M}^{i}$ ), if $\hat{x}^{i}<\delta_{1}^{i}\left(T, \bar{M}^{i}\right)$ and $\left|\hat{x}^{i}-x^{i}\right|^{2}<\delta_{2}^{i}\left(T, \bar{M}^{i}\right)$, one must have

$$
\mathbb{E}^{i}\left[\sup _{0 \leq t \leq T}\left|\widehat{X}^{i}(t)-X^{i}(t)\right|^{2}\right] \leq \varepsilon^{i},
$$


for $\forall i=1,2, \ldots, I$. Moreover, if we let $\lim _{T \rightarrow \infty} \delta_{j}^{i}\left(T, \bar{M}^{i}\right)=$ $0(j=1,2)$ for any given $\bar{M}^{i}(\forall i=1,2, \ldots, I)$, then one has

$$
\mathbb{E}^{i}\left[\lim _{T \rightarrow \infty} \sup _{0 \leq t \leq T}\left|\widehat{X}^{i}(t)-X^{i}(t)\right|^{2}\right]=0 .
$$

Notice, by Proposition 6, that the optimal consumption path amounts to

$$
\hat{c}^{i}(t)=\left[\left(1+\tau_{c}(t)\right) \gamma^{i} C^{i}(t)\right]^{1 /\left(\gamma^{i}-1\right)} \widehat{X}^{i}(t)=\widehat{\lambda}^{i}(t) \widehat{X}^{i}(t) .
$$

Thus, by Itô's rule and $\left(13^{\prime}\right)$, we get

$$
\begin{aligned}
d \widehat{c}^{i}(t)= & {\left[\widehat{\lambda}^{i \prime}(t) \widehat{X}^{i}(t)+\widehat{\lambda}^{i}(t) \widehat{f}^{i}\left(\widehat{X}^{i}(t)\right)\right] d t } \\
& +\widehat{\lambda}^{i}(t) \widehat{g}^{i}\left(\widehat{X}^{i}(t)\right) d W^{i}(t) \\
= & \widetilde{f}^{i}\left(\widehat{X}^{i}(t)\right) d t+\widetilde{g}^{i}\left(\widehat{X}^{i}(t)\right) d W^{i}(t),
\end{aligned}
$$

subject to $\hat{c}^{i}(0)=\hat{\lambda}^{i}(0) \hat{x}^{i}>0, \mathbb{P}^{i}$-a.s. for $\forall i=1,2, \ldots, I$. Moreover, when denoted by matrix form, we get

$$
d \widehat{c}(t)=\widetilde{f}(\widehat{X}(t)) d t+\widetilde{g}(\widehat{X}(t)) d W(t),
$$

subject to $\widehat{c}(0)=\operatorname{diag}\left(\widehat{\lambda}^{1}(0), \ldots, \hat{\lambda}^{I}(0)\right) \hat{x}>0, \mathbb{P}$-a.s..

In particular, if we are given the following case.

Case 1. There is a coefficient $\lambda^{i}(t)$ such that

$$
c^{i}(t)=\lambda^{i}(t) X^{i}(t), \quad t \geq 0,
$$

for $\forall i=1,2, \ldots, I$. That is, $c(t)=\operatorname{diag}\left(\lambda^{1}(t), \ldots, \lambda^{I}(t)\right) X(t)$ with $X(t)$ defined in (4) subject to $c(0)=\operatorname{diag}\left(\lambda^{1}(0)\right.$, $\left.\ldots, \lambda^{I}(0)\right) x>0, \mathbb{P}$-a.s.. ing.

Indeed, as corollaries of Theorem 14, we have the follow-

Corollary 17 (uniform topology golden rule). Based upon the assumptions and conclusions of Theorem 14, then for any given $T \geq 0, \forall \varepsilon \geq 0$, one gets

$$
\mathbb{E}\left[\sup _{0 \leq t \leq T}\|\widehat{c}(t)-c(t)\|_{2}^{2}\right] \leq \varepsilon .
$$

Moreover, similar to Theorem 14, one gets

$$
\mathbb{E}\left[\lim _{T \rightarrow \infty} \sup _{0 \leq t \leq T}\|\widehat{c}(t)-c(t)\|_{2}^{2}\right]=0 .
$$

Proof. See Appendix D.

If we define

$$
\Psi(t)=(c(t), X(t))^{\top}, \quad \widehat{\Psi}(t)=(\widehat{c}(t), \widehat{X}(t))^{\top} .
$$

Then we have the following.
Corollary 18 (uniform topology golden rule). Based upon the assumptions and conclusions of Theorem 14, then for any given $T \geq 0, \forall \varepsilon \geq 0$, one gets

$$
\mathbb{E}\left[\sup _{0 \leq t \leq T}\|\boldsymbol{\Psi}(t)-\widehat{\Psi}(t)\|_{2}^{2}\right] \leq \varepsilon .
$$

Moreover, similar to Theorem 14, one gets

$$
\mathbb{E}\left[\lim _{T \rightarrow \infty} \sup _{0 \leq t \leq T}\|\boldsymbol{\Psi}(t)-\widehat{\Psi}(t)\|_{2}^{2}\right]=0 .
$$

Proof. See Appendix E.

We now denote by

$$
\tau^{1}(t)=\left(\tau_{X}^{1}(t), \tau_{c}^{1}(t)\right), \quad \tau^{2}(t)=\left(\tau_{X}^{2}(t), \tau_{c}^{2}(t)\right)
$$

two alternative taxation policies. Then the corresponding optimal consumption paths are denoted by

$$
\begin{aligned}
& \widehat{c}\left(t, \tau^{1}(t)\right) \\
& \quad=\operatorname{diag}\left(\widehat{\lambda}^{1}\left(t, \tau^{1}(t)\right), \ldots, \widehat{\lambda}^{I}\left(t, \tau^{1}(t)\right)\right) \widehat{X}\left(t, \tau^{1}(t)\right), \\
& \widehat{c}\left(t, \tau^{2}(t)\right) \\
& \quad=\operatorname{diag}\left(\widehat{\lambda}^{1}\left(t, \tau^{2}(t)\right), \ldots, \hat{\lambda}^{I}\left(t, \tau^{2}(t)\right)\right) \widehat{X}\left(t, \tau^{2}(t)\right),
\end{aligned}
$$

respectively. Indeed, similar to Theorem 5 of Dai [43], we get the following.

Corollary 19 (inefficacy of temporary taxation policies). Based upon the assumptions and conclusions of Theorem 14, then for any given $T \geq 0, \forall \varepsilon \geq 0$, one gets

$$
\mathbb{E}\left[\sup _{0 \leq t \leq T}\left\|\widehat{c}\left(t, \tau^{1}(t)\right)-\widehat{c}\left(t, \tau^{2}(t)\right)\right\|_{2}^{2}\right] \leq \varepsilon .
$$

Moreover, similar to Theorem 14, one has

$$
\mathbb{E}\left[\lim _{T \rightarrow \infty} \sup _{0 \leq t \leq T}\left\|\widehat{c}\left(t, \tau^{1}(t)\right)-\widehat{c}\left(t, \tau^{2}(t)\right)\right\|_{2}^{2}\right]=0
$$

Proof. See Appendix F.

In (32), we suppose that there is a linear relationship between the original consumption path and the original wealth dynamics. Now, we relax this assumption and consider the following.

Case 2. We do not prespecify any relationship between consumption process $c(t)$ and wealth stock $X(t)$, which would be regarded as the most general case. 
We first introduce the following assumptions.

Assumption 20. There is a constant $H>0$ such that

$$
\|\widetilde{f}(\hat{y})\|_{2}^{2} \vee\|\widetilde{g}(\hat{y})\|_{2}^{2} \leq H^{2}\|\hat{y}\|_{2}^{2},
$$

for $\tilde{f}, \widetilde{g}$ defined in (31) and for $\forall \hat{y} \in \mathbb{R}_{+}^{I}$.

Assumption 21. For $\forall \varepsilon \geq 0$ and $\forall p \in \mathbb{N}, p \geq 2$, we have

$$
\mathbb{E}\|\widehat{c}(0)-c(0)\|_{2}^{p} \leq \varepsilon .
$$

Assumption 22. Consumption path $c(t)$ is continuously differentiable and for $\forall \varepsilon \geq 0, \forall p \in \mathbb{N}, p \geq 2$ and for any given $T \geq 0$, we get

$$
\int_{0}^{T} \mathbb{E}\left\|\nabla_{t} c(t)\right\|_{2}^{p} d t \leq \varepsilon
$$

where $\nabla_{t} c(t)=\left(c^{1 \prime}(t), \ldots, c^{I \prime}(t)\right)^{\top}$.

Consequently, we get the following theorem.

Theorem 23 (uniform topology golden rule). Based upon Assumptions 20, 21 and 22, and for $\forall p \in \mathbb{N}, p \geq 2$, any given $T \geq 0, \forall \varepsilon \geq 0$, one has

$$
\mathbb{E}\left[\sup _{0 \leq t \leq T}\|\widehat{c}(t)-c(t)\|_{2}^{p}\right] \leq \varepsilon .
$$

Moreover, one can show

$$
\mathbb{E}\left[\lim _{T \rightarrow \infty} \sup _{0 \leq t \leq T}\|\widehat{c}(t)-c(t)\|_{2}^{p}\right]=0 .
$$

Proof. See Appendix G.

Remark 24. One can easily tell the differences between Corollary 17 and Theorem 23, and indeed the main differences can be expressed as follows: first, Theorem 23 provides us with a much stronger conclusion than that of Corollary 17; second, noting that Theorem 23 corresponds to Case 2, which is much more general than Case 1, Corollary 17 depends on much weaker assumptions than Theorem 23. However, both Corollary 17 and Theorem 23 demonstrate the Liapounov stability and asymptotic stability of optimal consumption paths in the sense of uniform topology and in non-stationary environments.

\section{Concluding Remarks}

As is well known, theory about golden rule or modified golden rule and different types of turnpike theorems (i.e., Neighborhood Turnpike Theorem, see Yano [44], and Asymptotic Turnpike Theorem, see Yano [45]) have been developed and extensively studied for several decades and play very important roles in both macroeconomics and mathematical economics. Meanwhile, noting that portfolio choice, consumption strategy, and wealth accumulation are important issues in financial economics, especially in asset pricing models and market selection theories, and portfolio turnpikes has attracted broad interest of investigation while little attention has been focused on the turnpike or golden rule properties about consumption path and wealth dynamics in financial economics. Moreover, one can easily notice that portfolio choice, consumption strategy and wealth accumulation are intimately correlated with each other both in financial models and in financial markets. Accordingly, the current paper argues from the following two viewpoints that first, exploration about the golden rule or turnpike properties of consumption path and wealth accumulation should be of independent interest; second, the exploration of consumption path and wealth accumulation in different financial markets may possibly in turn enhance and deepen our understanding of portfolio turnpikes (see, [14]). And hence, the major purpose of the current paper is to meet the above gap and we indeed have proved the golden rules about consumption path and wealth dynamics in a type of complete financial markets with heterogeneous investors. Moreover, inefficacy of temporary taxation policies also has been confirmed in the present model, which would be regarded as a natural byproduct of the investigation about golden rules of the corresponding financial market.

Finally, the current paper can be naturally extended according to the following three lines: first, one can explore uniform topology turnpikes about consumption path and wealth accumulation in financial market, that is, one can search for the conditions under which the uniform topology golden rules demonstrated in the present paper are also uniform topology turnpikes; second, one can study the existence and uniqueness of uniform topology golden rule or turnpike of any given incomplete financial market or market with asymmetric information, that is, there exists insider trade in market, and to further characterize their properties from the view of point of economics; last but not least, one can, if motivated, investigate golden rules or turnpikes in the framework of general equilibrium or dynamic general equilibrium of any given financial market.

\section{Appendices}

\section{A. Proof of Proposition 6}

We do so by first defining the following process:

$$
Y^{i}(t)=\left(s+t, X^{i}(t)\right)^{\top}, \quad t \geq 0, Y^{i}\left(0^{-}\right)=\left(s, x^{i}\right)^{\top} .
$$

Thus, the differential generator of $Y^{i}(t)$ reads as follows:

$$
\begin{aligned}
\mathscr{L}^{i} \phi^{i}\left(s, x^{i}\right)=\frac{\partial \phi^{i}}{\partial s}+\{( & \left.1-\tau_{X}(0)\right) x^{i} \\
& \times\left[\pi^{i}(0)^{\top}\left(b^{i}(0)-r(0) \mathbf{1}\right)+r(0)\right] \\
& \left.-\left(1+\tau_{c}(0)\right) c^{i}(0)\right\} \frac{\partial \phi^{i}}{\partial x^{i}}
\end{aligned}
$$




$$
\begin{aligned}
& +\frac{1}{2}\left(1-\tau_{X}(0)\right)^{2} \pi^{i}(0)^{\top} \sigma(0) \sigma(0)^{\top} \\
& \times \pi^{i}(0)\left(x^{i}\right)^{2} \frac{\partial^{2} \phi^{i}}{\partial\left(x^{i}\right)^{2}} .
\end{aligned}
$$

If we try

$$
\phi^{i}\left(s, x^{i}\right)=e^{-\rho^{i} s} \varphi^{i}\left(x^{i}\right) .
$$

Then we get

$$
\begin{aligned}
& \mathscr{L}^{i} \phi^{i}\left(s, x^{i}\right) \\
& =e^{-\rho^{i} s}\left(-\rho^{i} \varphi^{i}\left(x^{i}\right)\right. \\
& +\left\{\left(1-\tau_{X}(0)\right)\right. \\
& \quad \times\left[\pi^{i}(0)^{\top}\left(b^{i}(0)-r(0) \mathbf{1}\right)+r(0)\right] x^{i} \\
& \left.\quad-\left(1+\tau_{c}(0)\right) c^{i}(0)\right\} \varphi^{i \prime}\left(x^{i}\right) \\
& +\frac{1}{2}\left(1-\tau_{X}(0)\right)^{2} \pi^{i}(0)^{\top} \sigma(0) \sigma(0)^{\top} \\
& \left.\times \pi^{i}(0)\left(x^{i}\right)^{2} \varphi^{i \prime \prime}\left(x^{i}\right)\right)=e^{-\rho^{i} s} \mathscr{L}_{0}^{i} \varphi^{i}\left(x^{i}\right),
\end{aligned}
$$

in particular, if we put

$$
\varphi^{i}\left(x^{i}\right)=C^{i}(0)\left(x^{i}\right)^{\gamma^{i}}
$$

then we get by the stochastic Hamilton-Jacobi-Bellman equation

$$
\begin{aligned}
\left(\hat{\pi}^{i}(0), \hat{c}^{i}(0)\right) & =\operatorname{argmax}\left\{\mathscr{L}_{0}^{i} \varphi^{i}\left(x^{i}\right)+\frac{c^{i}(0)^{\gamma^{i}}}{\gamma^{i}}\right\} \\
& =\operatorname{argmax}\left\{h^{i}\left(\pi^{i}(0), c^{i}(0)\right)\right\},
\end{aligned}
$$

where,

$$
\begin{aligned}
& h^{i}\left(\pi^{i}(0), c^{i}(0)\right) \\
&=- \rho^{i} C^{i}(0)\left(x^{i}\right)^{\gamma^{i}} \\
&+\left\{\left(1-\tau_{X}(0)\right)\left[\pi^{i}(0)^{\top}\left(b^{i}(0)-r(0) \mathbf{1}\right)+r(0)\right] x^{i}\right. \\
&\left.\quad\left(1+\tau_{c}(0)\right) c^{i}(0)\right\} C^{i}(0) \gamma^{i}\left(x^{i}\right)^{\gamma^{i}-1} \\
&+ \frac{1}{\gamma^{i}} c^{i}(0)^{\gamma^{i}}+\frac{1}{2}\left(1-\tau_{X}(0)\right)^{2} \pi^{i}(0)^{\top} \sigma(0)(0)^{\top} \\
& \times \pi^{i}(0) C^{i}(0) \gamma^{i}\left(\gamma^{i}-1\right)\left(x^{i}\right)^{\gamma^{i}} .
\end{aligned}
$$

Notice that $h^{i}$ is concave in $\left(\pi^{i}(0), c^{i}(0)\right)$, thus, the maximum of $h^{i}$ is attained via the following FOC:

$$
\begin{aligned}
\frac{\partial h^{i}}{\partial \pi^{i}}= & \left(1-\tau_{X}(0)\right)\left(b^{i}(0)-r(0) \mathbf{1}\right) C^{i}(0) \gamma^{i}\left(x^{i}\right)^{\gamma^{i}} \\
& +\left(1-\tau_{X}(0)\right)^{2} \sigma(0) \sigma(0)^{\top} \\
& \times \pi^{i}(0) C^{i}(0) \gamma^{i}\left(\gamma^{i}-1\right)\left(x^{i}\right)^{\gamma^{i}}=0, \\
\frac{\partial h^{i}}{\partial c^{i}}=- & \left(1+\tau_{c}(0)\right) C^{i}(0) \gamma^{i}\left(x^{i}\right)^{\gamma^{i}-1}+c^{i}(0)^{\gamma^{i}-1}=0 .
\end{aligned}
$$

Then one can easily get

$$
\begin{aligned}
\hat{\pi}^{i}(0)= & \frac{1}{\left(1-\tau_{X}(0)\right)\left(1-\gamma^{i}\right)} \\
& \times\left(\sigma(0) \sigma(0)^{\top}\right)^{-1}\left(b^{i}(0)-r(0) \mathbf{1}\right), \\
\hat{c}^{i}(0)= & {\left[\left(1+\tau_{c}(0)\right) \gamma^{i} C^{i}(0)\right]^{1 /\left(\gamma^{i}-1\right)} \widehat{X}^{i}(0), }
\end{aligned}
$$

noting that we should have

$$
h^{i}\left(\widehat{\pi}^{i}(0), \hat{c}^{i}(0)\right)=0
$$

thus, we get

$$
\begin{aligned}
C^{i}(0)= & \frac{\left(1-\gamma^{i}\right)^{1-\gamma^{i}}}{\gamma^{i}\left(1+\tau_{c}(0)\right)^{\gamma^{i}}} \\
& \times\left\{\rho^{i}-\left(1-\tau_{X}(0)\right)\right. \\
& \quad \times\left[\widehat{\pi}^{i}(0)^{\top}\left(b^{i}(0)-r(0) \mathbf{1}\right)+r(0)\right] \gamma^{i} \\
& +\frac{1}{2}\left(1-\tau_{X}(0)\right)^{2} \hat{\pi}^{i}(0)^{\top} \\
& \left.\quad \times \sigma(0) \sigma(0)^{\top} \hat{\pi}^{i}(0) \gamma^{i}\left(1-\gamma^{i}\right)\right\}^{\gamma^{i}-1}
\end{aligned}
$$

Consequently, applying the Markov properties of the solutions, the desired results in Proposition 6 are established.

\section{B. Proof of Lemma 10}

Applying Itô’s rule to (15), we get

$$
\begin{aligned}
\|\widehat{X}(t)\|_{2}^{2}= & \|\widehat{X}(0)\|_{2}^{2}+2 \int_{0}^{t}\langle\widehat{f}(\widehat{X}(s)), \widehat{X}(s)\rangle d s \\
& +\int_{0}^{t}\|\widehat{g}(\widehat{X}(s))\|_{2}^{2} d s \\
& +2 \int_{0}^{t}\langle\widehat{X}(s), \widehat{g}(\widehat{X}(s)) d W(s)\rangle,
\end{aligned}
$$


thus, for some constant $\widehat{K}=\widehat{K}(p, T)>0$, which may change at each occurrence throughout the proof, and $t_{1} \in[0, T]$, we obtain by using Assumption $5^{\prime}$

$$
\begin{aligned}
\sup _{0 \leq t \leq t_{1}}\|\widehat{X}(t)\|_{2}^{p} & \\
\leq & \widehat{K}\left\{\|\widehat{X}(0)\|_{2}^{p}+\left[\int_{0}^{t_{1}} \widehat{L}\|\widehat{X}(s)\|_{2}^{2} d s\right]^{p / 2}\right. \\
& \left.+\sup _{0 \leq t \leq t_{1}}\left|\int_{0}^{t}\langle\widehat{X}(s), \widehat{g}(\widehat{X}(s)) d W(s)\rangle\right|^{p / 2}\right\} .
\end{aligned}
$$

It follows from applying Cauchy-Schwarz inequality and taking expectations on both sides that

$$
\begin{aligned}
\mathbb{E}\left[\sup _{0 \leq t \leq t_{1}}\|\widehat{X}(t)\|_{2}^{p}\right] \\
\leq \widehat{K}\left\{\mathbb{E}\|\widehat{X}(0)\|_{2}^{p}+\mathbb{E}\left[\int_{0}^{t_{1}}\|\widehat{X}(s)\|_{2}^{p} d s\right]\right. \\
\left.+\mathbb{E}\left[\sup _{0 \leq t \leq t_{1}}\left|\int_{0}^{t}\langle\widehat{X}(s), \widehat{g}(\widehat{X}(s)) d W(s)\rangle\right|^{p / 2}\right]\right\} .
\end{aligned}
$$

Applying the Burkholder-Davis-Gundy inequality (see [46], pp. 166 ), we get

$$
\begin{aligned}
& \mathbb{E}\left[\sup _{0 \leq t \leq t_{1}}\|\widehat{X}(t)\|_{2}^{p}\right] \\
& \leq \widehat{K}\left\{\mathbb{E}\|\widehat{X}(0)\|_{2}^{p}+\int_{0}^{t_{1}} \mathbb{E}\|\widehat{X}(s)\|_{2}^{p} d s\right. \\
&\left.+\mathbb{E}\left[\int_{0}^{t_{1}}\|\widehat{X}(s)\|_{2}^{2}\|\widehat{g}(\widehat{X}(s))\|_{2}^{2} d s\right]^{p / 4}\right\} .
\end{aligned}
$$

Next, by using the Young inequality (see [47]), Assumption $5^{\prime}$, and Hölder inequality, we see that for $\widehat{K}$ given in (B.4)

$$
\begin{aligned}
& \mathbb{E}\left[\int_{0}^{t_{1}}\|\widehat{X}(s)\|_{2}^{2}\|\widehat{g}(\widehat{X}(s))\|_{2}^{2} d s\right]^{p / 4} \\
& \leq \mathbb{E}\left[\sup _{0 \leq t \leq t_{1}}\|\widehat{X}(t)\|_{2}^{p / 2}\left(\int_{0}^{t_{1}}\|\widehat{g}(\widehat{X}(s))\|_{2}^{2} d s\right)^{p / 4}\right] \\
& \leq \frac{1}{2 \widehat{K}} \mathbb{E}\left[\sup _{0 \leq t \leq t_{1}}\|\widehat{X}(t)\|_{2}^{p}\right]+\frac{\widehat{K}}{2}\left[\int_{0}^{t_{1}}\|\widehat{g}(\widehat{X}(s))\|_{2}^{2} d s\right]^{p / 2} \\
& \leq \frac{1}{2 \widehat{K}} \mathbb{E}\left[\sup _{0 \leq t \leq t_{1}}\|\widehat{X}(t)\|_{2}^{p}\right]+\frac{\widehat{K}}{2} \widehat{L}^{p / 2} \mathbb{E}\left[\int_{0}^{t_{1}}\|\widehat{X}(s)\|_{2}^{2} d s\right]^{p / 2} \\
& \leq \frac{1}{2 \widehat{K}} \mathbb{E}\left[\sup _{0 \leq t \leq t_{1}}\|\widehat{X}(t)\|_{2}^{p}\right]+\frac{\widehat{K}}{2} \widehat{L}^{p / 2} T^{(p-2) / 2} \mathbb{E}\left[\int_{0}^{t_{1}}\|\widehat{X}(s)\|_{2}^{p} d s\right] .
\end{aligned}
$$

Substituting this into (B.4) produces

$$
\mathbb{E}\left[\sup _{0 \leq t \leq T}\|\widehat{X}(t)\|_{2}^{p}\right] \leq \widehat{K}\left[\mathbb{E}\|\widehat{X}(0)\|_{2}^{p}+\int_{0}^{T} \mathbb{E}\|\widehat{X}(s)\|_{2}^{p} d s\right] .
$$

Thus, by applying Assumption 7 and the following fact (see, [47])

$$
\mathbb{E}\|\widehat{X}(t)\|_{2}^{p} \leq \widehat{K}(p, T)\left[1+\mathbb{E}\|\widehat{X}(0)\|_{2}^{p}\right]
$$

by directly applying (B.7) to the right hand side of inequality (B.6) to simplify the corresponding formula, which immediately gives rise to the desired assertion in the lemma.

\section{Proof of Theorem 14}

The idea of the proof comes from Higham et al. [47]. Provided Assumptions 2, $5^{\prime}$, and Condition 1, and given the wealth dynamics defined in (4) and (15), we get the corresponding strong solutions

$$
\begin{gathered}
X(t)=X(0)+\int_{0}^{t} f(X(s)) d s+\int_{0}^{t} g(X(s)) d W(s), \\
\widehat{X}(t)=\widehat{X}(0)+\int_{0}^{t} \widehat{f}(\widehat{X}(s)) d s+\int_{0}^{t} \widehat{g}(\widehat{X}(s)) d W(s),
\end{gathered}
$$

respectively. By Lemmas 10 and 12, we can choose some constant $M=e \vee \widehat{e}$ such that

$$
\mathbb{E}\left[\sup _{0 \leq t \leq T}\|X(t)\|_{2}^{p}\right] \vee \mathbb{E}\left[\sup _{0 \leq t \leq T}\|\widehat{X}(t)\|_{2}^{p}\right] \leq M
$$

using Assumption 2 and Condition 1, one can choose $\bar{M}=$ $R \vee \widehat{R}$ such that $\|\widehat{X}(t)\|_{2} \vee\|X(t)\|_{2} \leq \bar{M}, \forall t \geq 0$, otherwise we just consider $\widehat{X}(t) \wedge \mathbf{1}_{\bar{M}}$ and $X(t) \wedge \mathbf{1}_{\bar{M}}$ with $\mathbf{1}_{\bar{M}}=(\bar{M}, \bar{M}, \ldots, \bar{M})^{\top}$ instead of $\widehat{X}(t)$ and $X(t)$, respectively, and then send $\bar{M}$ into infinity by applying the well-known Lebesgue dominated convergence theorem. In what follows, we proceed by first defining the following stopping times:

$$
\begin{gathered}
\widehat{\tau}^{\bar{M}}=\inf \left\{t \geq 0 ;\|\widehat{X}(t)\|_{2} \geq \bar{M}\right\}, \\
\tau^{\bar{M}}=\inf \left\{t \geq 0 ;\|X(t)\|_{2} \geq \bar{M}\right\}, \\
\widetilde{\tau}^{\bar{M}}=\widehat{\tau}^{\bar{M}} \wedge \tau^{\bar{M}} .
\end{gathered}
$$


Then by the Young inequality (see, [47]) and for any $v>0$

$$
\begin{aligned}
& \mathbb{E}\left[\sup _{0 \leq t \leq T}\|\widehat{X}(t)-X(t)\|_{2}^{2}\right] \\
& =\mathbb{E}\left[\sup _{0 \leq t \leq T}\|\widehat{X}(t)-X(t)\|_{2}^{2} \chi_{\left\{\bar{\tau}^{\bar{M}}>T, \tau^{\bar{M}}>T\right\}}\right] \\
& +\mathbb{E}\left[\sup _{0 \leq t \leq T}\|\widehat{X}(t)-X(t)\|_{2}^{2} \chi_{\left\{\bar{\tau}^{\bar{M}} \leq T, \tau^{\bar{M}} \leq T\right\}}\right] \\
& \leq \mathbb{E}\left[\sup _{0 \leq t \leq T}\left\|\widehat{X}\left(t \wedge \widetilde{\tau}^{\bar{M}}\right)-X\left(t \wedge \widetilde{\tau}^{\bar{M}}\right)\right\|_{2}^{2} \chi_{\left\{\tilde{\tau}^{\bar{M}}>T\right\}}\right] \\
& +\frac{2 v}{p} \mathbb{E}\left[\sup _{0 \leq t \leq T}\|\widehat{X}(t)-X(t)\|_{2}^{p}\right] \\
& +\frac{1-(2 / p)}{v^{2 /(p-2)}} \mathbb{P}\left\{\widehat{\tau}^{\bar{M}} \leq T \text {, or } \tau^{\bar{M}} \leq T\right\},
\end{aligned}
$$

where $\chi_{\{\cdot\}}$ denotes indicator function of set $\{\cdot\}$. And it follows from (C.3) that

$$
\begin{aligned}
& \mathbb{P}\left\{\omega ; \widehat{\tau}^{\bar{M}}(\omega) \leq T\right\} \\
& =\mathbb{E}\left[\chi_{\left\{\bar{\tau}^{\bar{M}} \leq T\right\}} \frac{\left\|\widehat{X}\left(\widehat{\tau}^{M}\right)\right\|_{2}^{p}}{\bar{M}^{p}}\right] \leq \frac{1}{\bar{M}^{p}} \mathbb{E}\left[\sup _{0 \leq t \leq T}\|\widehat{X}(t)\|_{2}^{p}\right] \leq \frac{M}{\bar{M}^{p}} .
\end{aligned}
$$

And similarly, $\mathbb{P}\left\{\omega ; \tau^{\bar{M}}(\omega) \leq T\right\} \leq M / \bar{M}^{p}$. So we have

$$
\begin{aligned}
& \mathbb{P}\left\{\omega ; \widehat{\tau}^{\bar{M}}(\omega) \leq T, \text { or } \tau^{\bar{M}}(\omega) \leq T\right\} \\
& \quad \leq \mathbb{P}\left\{\omega ; \widehat{\tau}^{\bar{M}}(\omega) \leq T\right\}+\mathbb{P}\left\{\omega ; \tau^{\bar{M}}(\omega) \leq T\right\} \leq \frac{2 M}{\bar{M}^{p}} .
\end{aligned}
$$

Thus, we obtain

$$
\begin{aligned}
& \mathbb{E}\left[\sup _{0 \leq t \leq T}\|\widehat{X}(t)-X(t)\|_{2}^{p}\right] \\
& \quad \leq 2^{p-1} \mathbb{E}\left[\sup _{0 \leq t \leq T}\left(\|\widehat{X}(t)\|_{2}^{p}+\|X(t)\|_{2}^{p}\right)\right] \leq 2^{p} M .
\end{aligned}
$$

Hence, (C.5) becomes

$$
\begin{gathered}
\mathbb{E}\left[\sup _{0 \leq t \leq T}\|\widehat{X}(t)-X(t)\|_{2}^{2}\right] \\
\leq \mathbb{E}\left[\sup _{0 \leq t \leq T}\left\|\widehat{X}\left(t \wedge \widetilde{\tau}^{\bar{M}}\right)-X\left(t \wedge \widetilde{\tau}^{\bar{M}}\right)\right\|_{2}^{2}\right] \\
+\frac{2^{p+1} v M}{p}+\frac{2(p-2) M}{p v^{2 /(p-2)} \bar{M}^{p}} .
\end{gathered}
$$

Thus, by using Cauchy-Schwarz inequality and Assumptions 2 and 13 , we have

$$
\begin{aligned}
& \left\|\widehat{X}\left(t \wedge \widetilde{\tau}^{\bar{M}}\right)-X\left(t \wedge \widetilde{\tau}^{\bar{M}}\right)\right\|_{2}^{2} \\
& =\|[\widehat{X}(0)-X(0)]+\int_{0}^{t \wedge \widetilde{\tau}^{\bar{M}}}[\widehat{f}(\widehat{X}(s))-f(X(s))] d s \\
& +\int_{0}^{t \wedge \widetilde{\tau}^{\bar{M}}}[\widehat{g}(\widehat{X}(s))-g(X(s))] d W(s) \|_{2}^{2} \\
& \leq 2\left[\|\widehat{X}(0)-X(0)\|_{2}^{2}\right. \\
& +T \int_{0}^{t \wedge \widetilde{\tau}^{\bar{M}}}\|\widehat{f}(\widehat{X}(s))-f(X(s))\|_{2}^{2} d s \\
& \left.+\left\|\int_{0}^{t \wedge \bar{\tau}^{\bar{M}}}[\widehat{g}(\widehat{X}(s))-g(X(s))] d W(s)\right\|_{2}^{2}\right] .
\end{aligned}
$$

Taking expectation on both sides and applying the Itô isometry reveals

$$
\begin{aligned}
\mathbb{E}\left[\left\|\widehat{X}\left(t \wedge \widetilde{\tau}^{\bar{M}}\right)-X\left(t \wedge \widetilde{\tau}^{\bar{M}}\right)\right\|_{2}^{2}\right] \\
\leq 4 \mathbb{E}\left[\|\widehat{X}(0)-X(0)\|_{2}^{2}\right. \\
+T \int_{0}^{t \wedge \widetilde{\tau}^{\bar{M}}}\|f(X(s))-f(\widehat{X}(s))\|_{2}^{2} d s \\
+T \int_{0}^{t \wedge \widetilde{\tau}^{\bar{M}}}\|f(\widehat{X}(s))-\widehat{f}(\widehat{X}(s))\|_{2}^{2} d s \\
+\int_{0}^{t \wedge \widetilde{\tau}^{\bar{M}}}\|g(X(s))-g(\widehat{X}(s))\|_{2}^{2} d s \\
\left.+\int_{0}^{t \wedge \widetilde{\tau}^{\bar{M}}}\|g(\widehat{X}(s))-\widehat{g}(\widehat{X}(s))\|_{2}^{2} d s\right]
\end{aligned}
$$

$$
\begin{aligned}
\leq 4 \mathbb{E}\left[\|\widehat{X}(0)-X(0)\|_{2}^{2}+T L_{R} \int_{0}^{t \wedge \widetilde{\tau}^{\bar{M}}}\|X(s)-\widehat{X}(s)\|_{2}^{2} d s\right. \\
+T K \int_{0}^{t \wedge \widetilde{\tau}^{\bar{M}}}\|\widehat{X}(s)\|_{2}^{2} d s+L_{R} \int_{0}^{t \wedge \widetilde{\tau}^{\bar{M}}}\|X(s)-\widehat{X}(s)\|_{2}^{2} d s \\
\left.+K \int_{0}^{t \wedge \widetilde{\tau}^{\bar{M}}}\|\widehat{X}(s)\|_{2}^{2} d s\right] .
\end{aligned}
$$


Thus, by Assumptions 1 and 7, for any $\tau \leq T$, we have

$$
\begin{aligned}
\mathbb{E}\left[\sup _{0 \leq t \leq \tau}\left\|\widehat{X}\left(t \wedge \widetilde{\tau}^{M}\right)-X\left(t \wedge \widetilde{\tau}^{M}\right)\right\|_{2}^{2}\right] \\
\leq 4\left[\mathbb{E}\|\widehat{X}(0)-X(0)\|_{2}^{2}+(T+1) L_{R} \mathbb{E}\right. \\
\quad \times \int_{0}^{t \wedge \widetilde{\tau}^{\bar{M}}}\|X(s)-\widehat{X}(s)\|_{2}^{2} d s \\
\left.\quad+(T+1) K \int_{0}^{t \wedge \widetilde{\tau}^{\bar{M}}} \mathbb{E}\|\widehat{X}(s)\|_{2}^{2} d s\right] \\
\leq 4\|\widehat{X}(0)-X(0)\|_{2}^{2}+4(T+1) L_{R} \\
\times \int_{0}^{\tau} \mathbb{E}\left[\sup _{0 \leq t_{0} \leq s}\left\|\widehat{X}\left(t_{0} \wedge \widetilde{\tau}^{M}\right)-X\left(t_{0} \wedge \widetilde{\tau}^{M}\right)\right\|_{2}^{2}\right] d s \\
+4(T+1) K \int_{0}^{T} \mathbb{E}\|\widehat{X}(s)\|_{2}^{2} d s .
\end{aligned}
$$

So the Gronwall's inequality (see, [47]) yields

$$
\begin{aligned}
& \mathbb{E}\left[\sup _{0 \leq t \leq \tau}\left\|\widehat{X}\left(t \wedge \widetilde{\tau}^{\bar{M}}\right)-X\left(t \wedge \bar{\tau}^{\bar{M}}\right)\right\|_{2}^{2}\right] \\
& \leq 4\left[\|\widehat{X}(0)-X(0)\|_{2}^{2}+(T+1) K \int_{0}^{T} \mathbb{E}\|\widehat{X}(s)\|_{2}^{2} d s\right] \\
& \quad \times e^{4(T+1) L_{R}} .
\end{aligned}
$$

Inserting this into (C.9) gives

$$
\begin{aligned}
\mathbb{E}\left[\sup _{0 \leq t \leq T}\|\widehat{X}(t)-X(t)\|_{2}^{2}\right] \\
\leq 4\left[\|\widehat{X}(0)-X(0)\|_{2}^{2}+(T+1) K \int_{0}^{T} \mathbb{E}\|\widehat{X}(s)\|_{2}^{2} d s\right] \\
\quad \times e^{4(T+1) L_{R}}+\frac{2^{p+1} v M}{p}+\frac{2(p-2) M}{p v^{2 /(p-2)} \bar{M}^{p}} .
\end{aligned}
$$

Hence, for $\forall \varepsilon>0$, we can choose some $v$ and $\bar{M}$ such that

$$
\frac{2^{p+1} v M}{p} \leq \frac{\varepsilon}{4}, \quad \frac{2(p-2) M}{p v^{2 /(p-2)} \bar{M}^{p}} \leq \frac{\varepsilon}{4} .
$$

Noting that $\widehat{X}(t)$, defined in (15), is a vector of Geometric Brownian motions, which combines with Assumption 7 shows that for any given $T>0$ and $\bar{M}>0$, there is a $\delta_{1}(T, \bar{M})>0$ such that if $\vee_{i=1}^{I} \widehat{x}^{i}<\delta_{1}(T, \bar{M})$, then we have

$$
4 e^{4(T+1) L_{R}}(T+1) K \int_{0}^{T} \mathbb{E}\|\widehat{X}(s)\|_{2}^{2} d s \leq \frac{\varepsilon}{4} .
$$

Moreover, we can choose $\delta_{2}(T, \bar{M})>0$ such that if $\|\widehat{x}-x\|_{2}^{2}<$ $\delta_{2}(T, \bar{M})$, then we have

$$
4 e^{4(T+1) L_{R}}\|\widehat{X}(0)-X(0)\|_{2}^{2} \leq \frac{\varepsilon}{4} .
$$

To summarize, we obtain the desired results in the theorem, that is,

$$
\mathbb{E}\left[\sup _{0 \leq t \leq T}\|\widehat{X}(t)-X(t)\|_{2}^{2}\right] \leq \varepsilon
$$

Moreover, noting that, for any given $\bar{M}, \lim _{T \rightarrow \infty} \delta_{i}(T, \bar{M})=$ $0(\forall i=1,2)$, thus, we have

$$
\lim _{T \rightarrow \infty} \mathbb{E}\left[\sup _{0 \leq t \leq T}\|\widehat{X}(t)-X(t)\|_{2}^{2}\right]=0 .
$$

Applying the well-known Levi lemma leads us to

$$
\mathbb{E}\left[\lim _{T \rightarrow \infty} \sup _{0 \leq t \leq T}\|\widehat{X}(t)-X(t)\|_{2}^{2}\right]=0,
$$

which completes the proof.

\section{Proof of Corollary 17}

By (29), we get

$$
\widehat{c}(t)=\operatorname{diag}\left(\widehat{\lambda}^{1}(t), \ldots, \widehat{\lambda}^{I}(t)\right) \widehat{X}(t),
$$

and we put

$$
\widehat{\lambda}(t)=\left(\widehat{\lambda}^{1}(t), \ldots, \hat{\lambda}^{I}(t)\right)^{\top} .
$$

Similarly, by (32), we obtain

$$
c(t)=\operatorname{diag}\left(\lambda^{1}(t), \ldots, \lambda^{I}(t)\right) X(t),
$$

and we put

$$
\lambda(t)=\left(\lambda^{1}(t), \ldots, \lambda^{I}(t)\right)^{\top} .
$$


Thus, we get

$$
\begin{aligned}
& \|c(t)-\widehat{c}(t)\|_{2}^{2} \\
& =\left\|\operatorname{diag}\left(\lambda^{1}(t), \ldots, \lambda^{I}(t)\right) X(t)-\operatorname{diag}\left(\widehat{\lambda}^{1}(t), \ldots, \widehat{\lambda}^{I}(t)\right) \widehat{X}(t)\right\|_{2}^{2} \\
& =\| \operatorname{diag}\left(\lambda^{1}(t), \ldots, \lambda^{I}(t)\right) X(t) \\
& \quad-\operatorname{diag}\left(\lambda^{1}(t), \ldots, \lambda^{I}(t)\right) \widehat{X}(t) \\
& \quad+\operatorname{diag}\left(\lambda^{1}(t), \ldots, \lambda^{I}(t)\right) \widehat{X}(t) \\
& \quad-\operatorname{diag}\left(\widehat{\lambda}^{1}(t), \ldots, \hat{\lambda}^{I}(t)\right) \widehat{X}(t) \|_{2}^{2} \\
& \leq 2\left\|\operatorname{diag}\left(\lambda^{1}(t), \ldots, \lambda^{I}(t)\right)(X(t)-\widehat{X}(t))\right\|_{2}^{2} \\
& \quad+2\left\|\operatorname{diag}\left(\lambda^{1}(t)-\widehat{\lambda}^{1}(t), \ldots, \lambda^{I}(t)-\widehat{\lambda}^{I}(t)\right) \widehat{X}(t)\right\|_{2}^{2} \\
& \leq 2\left\|\operatorname{diag}\left(\lambda^{1}(t), \ldots, \lambda^{I}(t)\right)\right\|_{2}^{2}\|X(t)-\widehat{X}(t)\|_{2}^{2} \\
& \quad+2\left\|\operatorname{diag}\left(\lambda^{1}(t)-\widehat{\lambda}^{1}(t), \ldots, \lambda^{I}(t)-\widehat{\lambda}^{I}(t)\right)\right\|_{2}^{2}\|\widehat{X}(t)\|_{2}^{2} \\
& =2\|\lambda(t)\|_{2}^{2}\|X(t)-\widehat{X}(t)\|_{2}^{2}+2\|\lambda(t)-\widehat{\lambda}(t)\|_{2}^{2}\|\widehat{X}(t)\|_{2}^{2} .
\end{aligned}
$$

Noting that

$$
\begin{gathered}
\|\lambda(t)\|_{2}^{2} \leq I, \\
\|\lambda(t)-\hat{\lambda}(t)\|_{2}^{2} \leq 2\left(\|\lambda(t)\|_{2}^{2}+\|\hat{\lambda}(t)\|_{2}^{2}\right) \leq 4 I .
\end{gathered}
$$

Then, we have

$$
\|c(t)-\widehat{c}(t)\|_{2}^{2} \leq 2 I\|X(t)-\widehat{X}(t)\|_{2}^{2}+8 I\|\widehat{X}(t)\|_{2}^{2},
$$

hence, we get

$$
\begin{aligned}
& \mathbb{E}\left[\sup _{0 \leq t \leq T}\|c(t)-\widehat{c}(t)\|_{2}^{2}\right] \\
& \leq 2 I \mathbb{E}\left[\sup _{0 \leq t \leq T}\|X(t)-\widehat{X}(t)\|_{2}^{2}\right]+8 I \mathbb{E}\left[\sup _{0 \leq t \leq T}\|\widehat{X}(t)\|_{2}^{2}\right] .
\end{aligned}
$$

Based on the assumptions and conclusions of Theorem 14, one can show that, for $\forall \varepsilon>0$,

$$
\begin{gathered}
\mathbb{E}\left[\sup _{0 \leq t \leq T}\|X(t)-\widehat{X}(t)\|_{2}^{2}\right] \leq \frac{\varepsilon}{4 I}, \\
\mathbb{E}\left[\sup _{0 \leq t \leq T}\|\widehat{X}(t)\|_{2}^{2}\right] \leq \frac{\varepsilon}{16 I} .
\end{gathered}
$$

To sum up, we have

$$
\mathbb{E}\left[\sup _{0 \leq t \leq T}\|c(t)-\widehat{c}(t)\|_{2}^{2}\right] \leq \frac{\varepsilon}{2}+\frac{\varepsilon}{2}=\varepsilon .
$$

Furthermore, similar to the proof of Theorem 14, we also get by using Levi lemma

$$
\mathbb{E}\left[\lim _{T \rightarrow \infty} \sup _{0 \leq t \leq T}\|c(t)-\widehat{c}(t)\|_{2}^{2}\right]=0 .
$$

This completes the proof.

\section{E. Proof of Corollary 18}

Noting that

$$
\begin{aligned}
& \Psi(t)=\left(\begin{array}{c}
c(t) \\
X(t)
\end{array}\right)=\left(\begin{array}{c}
\operatorname{diag}\left(\lambda^{1}(t), \ldots, \lambda^{I}(t)\right) \\
\mathbf{I}_{I \times I}
\end{array}\right) X(t) \\
& =\mathbf{A} X(t), \\
& \widehat{\Psi}(t)=\left(\begin{array}{c}
\widehat{c}(t) \\
\widehat{X}(t)
\end{array}\right)=\left(\begin{array}{c}
\operatorname{diag}\left(\widehat{\lambda}^{1}(t), \ldots, \hat{\lambda}^{I}(t)\right) \\
\mathbf{I}_{I \times I}
\end{array}\right) \widehat{X}(t) \\
& =\widehat{\mathbf{A}} \widehat{X}(t) .
\end{aligned}
$$

Thus,

$$
\begin{aligned}
&\|\boldsymbol{\Psi}(t)-\widehat{\Psi}(t)\|_{2}^{2} \\
&=\|\mathbf{A} X(t)-\widehat{\mathbf{A}} \widehat{X}(t)\|_{2}^{2} \\
&=\|\mathbf{A} X(t)-\mathbf{A} \widehat{X}(t)+\mathbf{A} \widehat{X}(t)-\widehat{\mathbf{A}} \widehat{X}(t)\|_{2}^{2} \\
& \leq 2\|\mathbf{A}(X(t)-\widehat{X}(t))\|_{2}^{2}+2\|(\mathbf{A}-\widehat{\mathbf{A}}) \widehat{X}(t)\|_{2}^{2} \\
& \leq 2\|\mathbf{A}\|_{2}^{2}\|X(t)-\widehat{X}(t)\|_{2}^{2}+2\|\mathbf{A}-\widehat{\mathbf{A}}\|_{2}^{2}\|\widehat{X}(t)\|_{2}^{2} \\
&= 2\left(\|\lambda(t)\|_{2}^{2}+I\right)\|X(t)-\widehat{X}(t)\|_{2}^{2} \\
&+2\|\lambda(t)-\widehat{\lambda}(t)\|_{2}^{2}\|\widehat{X}(t)\|_{2}^{2} \\
& \leq 4 I\|X(t)-\widehat{X}(t)\|_{2}^{2}+8 I\|\widehat{X}(t)\|_{2}^{2} .
\end{aligned}
$$

Then we have

$$
\begin{aligned}
& \mathbb{E}\left[\sup _{0 \leq t \leq T}\|\Psi(t)-\widehat{\Psi}(t)\|_{2}^{2}\right] \\
& \leq 4 I \mathbb{E}\left[\sup _{0 \leq t \leq T}\|X(t)-\widehat{X}(t)\|_{2}^{2}\right]+8 I \mathbb{E}\left[\sup _{0 \leq t \leq T}\|\widehat{X}(t)\|_{2}^{2}\right] .
\end{aligned}
$$

Based upon the assumptions and conclusions of Theorem 14, one can choose conditions such that, for $\forall \varepsilon>0$,

$$
\begin{gathered}
\mathbb{E}\left[\sup _{0 \leq t \leq T}\|X(t)-\widehat{X}(t)\|_{2}^{2}\right] \leq \frac{\varepsilon}{8 I}, \\
\mathbb{E}\left[\sup _{0 \leq t \leq T}\|\widehat{X}(t)\|_{2}^{2}\right] \leq \frac{\varepsilon}{16 I} .
\end{gathered}
$$

Therefore, we get

$$
\mathbb{E}\left[\sup _{0 \leq t \leq T}\|\Psi(t)-\widehat{\Psi}(t)\|_{2}^{2}\right] \leq \frac{\varepsilon}{2}+\frac{\varepsilon}{2}=\varepsilon .
$$


Moreover, similar to the proof of Theorem 14, we get by applying the well-known Levi lemma

$$
\mathbb{E}\left[\lim _{T \rightarrow \infty} \sup _{0 \leq t \leq T}\|\boldsymbol{\Psi}(t)-\widehat{\Psi}(t)\|_{2}^{2}\right]=0,
$$

which gives the desired result.

\section{F. Proof of Corollary 19}

We define

$$
\begin{aligned}
& \hat{\lambda}\left(t, \tau^{1}(t)\right)=\left(\hat{\lambda}^{1}\left(t, \tau^{1}(t)\right), \ldots, \hat{\lambda}^{I}\left(t, \tau^{1}(t)\right)\right)^{\top}, \\
& \hat{\lambda}\left(t, \tau^{2}(t)\right)=\left(\hat{\lambda}^{1}\left(t, \tau^{2}(t)\right), \ldots, \hat{\lambda}^{I}\left(t, \tau^{2}(t)\right)\right)^{\top} .
\end{aligned}
$$

Then we get

$$
\begin{aligned}
\left\|\widehat{c}\left(t, \tau^{1}(t)\right)-\widehat{c}\left(t, \tau^{2}(t)\right)\right\|_{2}^{2} & \| \operatorname{diag}\left(\widehat{\lambda}^{1}\left(t, \tau^{1}(t)\right), \ldots, \widehat{\lambda}^{I}\left(t, \tau^{1}(t)\right)\right) \widehat{X}\left(t, \tau^{1}(t)\right) \\
& -\operatorname{diag}\left(\widehat{\lambda}^{1}\left(t, \tau^{2}(t)\right), \ldots, \widehat{\lambda}^{I}\left(t, \tau^{2}(t)\right)\right) \widehat{X}\left(t, \tau^{2}(t)\right) \|_{2}^{2} \\
\leq & 2\left\|\operatorname{diag}\left(\widehat{\lambda}^{1}\left(t, \tau^{1}(t)\right), \ldots, \hat{\lambda}^{I}\left(t, \tau^{1}(t)\right)\right) \widehat{X}\left(t, \tau^{1}(t)\right)\right\|_{2}^{2} \\
& +2\left\|\operatorname{diag}\left(\widehat{\lambda}^{1}\left(t, \tau^{2}(t)\right), \ldots, \widehat{\lambda}^{I}\left(t, \tau^{2}(t)\right)\right) \widehat{X}\left(t, \tau^{2}(t)\right)\right\|_{2}^{2} \\
\leq & 2\left\|\operatorname{diag}\left(\widehat{\lambda}^{1}\left(t, \tau^{1}(t)\right), \ldots, \widehat{\lambda}^{I}\left(t, \tau^{1}(t)\right)\right)\right\|_{2}^{2}\left\|\widehat{X}\left(t, \tau^{1}(t)\right)\right\|_{2}^{2} \\
& +2\left\|\operatorname{diag}\left(\widehat{\lambda}^{1}\left(t, \tau^{2}(t)\right), \ldots, \widehat{\lambda}^{I}\left(t, \tau^{2}(t)\right)\right)\right\|_{2}^{2}\left\|\widehat{X}\left(t, \tau^{2}(t)\right)\right\|_{2}^{2} \\
= & 2\left\|\widehat{\lambda}\left(t, \tau^{1}(t)\right)\right\|_{2}^{2}\left\|\widehat{X}\left(t, \tau^{1}(t)\right)\right\|_{2}^{2} \\
& +2\left\|\widehat{\lambda}\left(t, \tau^{2}(t)\right)\right\|_{2}^{2}\left\|\widehat{X}\left(t, \tau^{2}(t)\right)\right\|_{2}^{2} \\
\leq & 2 I\left(\left\|\widehat{X}\left(t, \tau^{1}(t)\right)\right\|_{2}^{2}+\left\|\widehat{X}\left(t, \tau^{2}(t)\right)\right\|_{2}^{2}\right) .
\end{aligned}
$$

Thus, based upon the assumptions similar to Theorem 14, for $\forall \varepsilon>0$, there exist $\delta_{1}(T), \delta_{2}(T)>0$ such that if $\vee_{i=1}^{I} \widehat{X}\left(0, \tau^{1}(0)\right)<\delta_{1}(T)$ and $\vee_{i=1}^{I} \widehat{X}\left(0, \tau^{2}(0)\right)<\delta_{2}(T)$, then we have

$$
\left\|\widehat{X}\left(t, \tau^{1}(t)\right)\right\|_{2}^{2} \leq \frac{\varepsilon}{4 I}, \quad\left\|\widehat{X}\left(t, \tau^{2}(t)\right)\right\|_{2}^{2} \leq \frac{\varepsilon}{4 I} .
$$

To summarize, we get

$$
\left\|\widehat{c}\left(t, \tau^{1}(t)\right)-\widehat{c}\left(t, \tau^{2}(t)\right)\right\|_{2}^{2} \leq \frac{\varepsilon}{2}+\frac{\varepsilon}{2}=\varepsilon, \quad \forall 0 \leq t \leq T .
$$

Then we obtain

$$
\mathbb{E}\left[\sup _{0 \leq t \leq T}\left\|\widehat{c}\left(t, \tau^{1}(t)\right)-\widehat{c}\left(t, \tau^{2}(t)\right)\right\|_{2}^{2}\right] \leq \varepsilon .
$$

Moreover, similar to the assumption and proof of Theorem 14, we get by using the well-known Levi lemma

$$
\mathbb{E}\left[\lim _{T \rightarrow \infty} \sup _{0 \leq t \leq T}\left\|\widehat{c}\left(t, \tau^{1}(t)\right)-\widehat{c}\left(t, \tau^{2}(t)\right)\right\|_{2}^{2}\right]=0,
$$

which completes the proof.

\section{G. Proof of Theorem 23}

By using Itô's rule and (31), one gets

$$
\begin{aligned}
\|\widehat{c}(t)-c(t)\|_{2}^{2}= & \|\widehat{c}(0)-c(0)\|_{2}^{2} \\
& +2 \int_{0}^{t}\left\langle c(s)-\widehat{c}(s), \nabla_{s} c(s)\right\rangle d s \\
& +2 \int_{0}^{t}\langle\widehat{c}(s)-c(s), \tilde{f}(\widehat{X}(s))\rangle d s \\
& +\int_{0}^{t}\|\widetilde{g}(\widehat{X}(s))\|_{2}^{2} d s \\
& +2 \int_{0}^{t}\langle\widehat{c}(s)-c(s), \widetilde{g}(\widehat{X}(s)) d W(s)\rangle .
\end{aligned}
$$

Then, by applying Assumption 20, for some constant $\xi=$ $\xi(p, T)>0$, which may be different from line to line throughout the proof, and for $t_{1} \in[0, T]$

$$
\begin{aligned}
& \sup _{0 \leq t \leq t_{1}}\|\widehat{c}(t)-c(t)\|_{2}^{p} \\
& \leq \xi\left\{\|\widehat{c}(0)-c(0)\|_{2}^{p}+H^{p}\left[\int_{0}^{t_{1}}\|\widehat{X}(s)\|_{2}^{2} d s\right]^{p / 2}\right. \\
& +\left[\int_{0}^{t_{1}}\left|\left\langle c(s)-\widehat{c}(s), \nabla_{s} c(s)\right\rangle\right| d s\right]^{p / 2} \\
& +\left[\int_{0}^{t_{1}}|\langle c(s)-\widehat{c}(s), \tilde{f}(\widehat{X}(s))\rangle| d s\right]^{p / 2} \\
& \left.+\sup _{0 \leq t \leq t_{1}}\left|\int_{0}^{t}\langle\widehat{c}(s)-c(s), \widetilde{g}(\widehat{X}(s)) d W(s)\rangle\right|^{p / 2}\right\} .
\end{aligned}
$$

It follows from Cauchy-Schwarz inequality that

$$
\begin{aligned}
& \sup _{0 \leq t \leq t_{1}}\|\widehat{c}(t)-c(t)\|_{2}^{p} \\
& \leq \xi\left\{\|\widehat{c}(0)-c(0)\|_{2}^{p}+\left[\int_{0}^{t_{1}}\|c(s)-\widehat{c}(s)\|_{2}\left\|\nabla_{s} c(s)\right\|_{2} d s\right]^{p / 2}\right. \\
& +\int_{0}^{t_{1}}\|\widehat{X}(s)\|_{2}^{p} d s \\
& +\left[\int_{0}^{t_{1}}\|c(s)-\widehat{c}(s)\|_{2}\|\widetilde{f}(\widehat{X}(s))\|_{2} d s\right]^{p / 2} \\
& \left.\quad+\sup _{0 \leq t \leq t_{1}}\left|\int_{0}^{t}\langle\widehat{c}(s)-c(s), \widetilde{g}(\widehat{X}(s)) d W(s)\rangle\right|^{p / 2}\right\} .
\end{aligned}
$$


By Young inequality (see, [47]), we get

$$
\begin{aligned}
& {\left[\int_{0}^{t_{1}}\|c(s)-\widehat{c}(s)\|_{2}\left\|\nabla_{s} c(s)\right\|_{2} d s\right]^{p / 2}} \\
& \leq \sup _{0 \leq t \leq t_{1}}\|c(t)-\widehat{c}(t)\|_{2}^{p / 2}\left[\int_{0}^{t_{1}}\left\|\nabla_{s} c(s)\right\|_{2} d s\right]^{p / 2} \\
& \leq \frac{1}{4 \xi} \sup _{0 \leq t \leq t_{1}}\|c(t)-\widehat{c}(t)\|_{2}^{p}+\xi\left[\int_{0}^{t_{1}}\left\|\nabla_{s} c(s)\right\|_{2} d s\right]^{p} .
\end{aligned}
$$

Similarly, using Young inequality (see, [47]) and Assumption 20, we obtain

$$
\begin{aligned}
& {\left[\int_{0}^{t_{1}}\|c(s)-\widehat{c}(s)\|_{2}\|\tilde{f}(\widehat{X}(s))\|_{2} d s\right]^{p / 2}} \\
& \leq \frac{1}{4 \xi} \sup _{0 \leq t \leq t_{1}}\|c(t)-\widehat{c}(t)\|_{2}^{p}+\xi H^{p}\left[\int_{0}^{t_{1}}\|\widehat{X}(s)\|_{2} d s\right]^{p} .
\end{aligned}
$$

Substituting (G.4) and (G.5) into (G.3) gives

$$
\begin{aligned}
& \sup _{0 \leq t \leq t_{1}}\|\widehat{c}(t)-c(t)\|_{2}^{p} \\
& \leq \xi\left\{\|\widehat{c}(0)-c(0)\|_{2}^{p}+\left[\int_{0}^{t_{1}}\left\|\nabla_{s} c(s)\right\|_{2} d s\right]^{p}\right. \\
& \quad+\int_{0}^{t_{1}}\|\widehat{X}(s)\|_{2}^{p} d s+\left[\int_{0}^{t_{1}}\|\widehat{X}(s)\|_{2} d s\right]^{p} \\
& \left.\quad+\sup _{0 \leq t \leq t_{1}}\left|\int_{0}^{t}\langle\widehat{c}(s)-c(s), \tilde{g}(\widehat{X}(s)) d W(s)\rangle\right|^{p / 2}\right\} .
\end{aligned}
$$

Taking expectations on both sides and applying BurkholderDavis-Gundy inequality (see, [46], pp.166) show

$$
\begin{gathered}
\mathbb{E}\left[\sup _{0 \leq t \leq t_{1}}\|\widehat{c}(t)-c(t)\|_{2}^{p}\right] \\
\leq \xi\left\{\mathbb{E}\|\widehat{c}(0)-c(0)\|_{2}^{p}+\mathbb{E}\left[\int_{0}^{t_{1}}\left\|\nabla_{s} c(s)\right\|_{2} d s\right]^{p}\right. \\
+\int_{0}^{t_{1}} \mathbb{E}\|\widehat{X}(s)\|_{2}^{p} d s+\mathbb{E}\left[\int_{0}^{t_{1}}\|\widehat{X}(s)\|_{2} d s\right]^{p} \\
\left.+\mathbb{E}\left[\int_{0}^{t_{1}}\|\widehat{c}(s)-c(s)\|_{2}^{2}\|\tilde{g}(\widehat{X}(s))\|_{2}^{2} d s\right]^{p / 4}\right\} .
\end{gathered}
$$

Next, by using the Young inequality (see, [47]), Hölder inequality, and Assumption 20, we see that

$$
\begin{aligned}
& \mathbb{E}\left[\int_{0}^{t_{1}}\|\widehat{c}(s)-c(s)\|_{2}^{2}\|\widetilde{g}(\widehat{X}(s))\|_{2}^{2} d s\right]^{p / 4} \\
& \leq \mathbb{E}\left[\sup _{0 \leq t \leq t_{1}}\|c(t)-\widehat{c}(t)\|_{2}^{p / 2}\left(\int_{0}^{t_{1}}\|\widetilde{g}(\widehat{X}(s))\|_{2}^{2} d s\right)^{p / 4}\right] \\
& \leq \frac{1}{2 \xi} \mathbb{E}\left[\sup _{0 \leq t \leq t_{1}}\|c(t)-\widehat{c}(t)\|_{2}^{p}\right]+\frac{\xi}{2} H^{p} \mathbb{E}\left[\int_{0}^{t_{1}}\|\widehat{X}(s)\|_{2}^{2} d s\right]^{p / 2} \\
& \leq \frac{1}{2 \xi} \mathbb{E}\left[\sup _{0 \leq t \leq t_{1}}\|c(t)-\widehat{c}(t)\|_{2}^{p}\right] \\
& \quad+\frac{\xi}{2} H^{p} T^{(p-2) / 2} \mathbb{E}\left[\int_{0}^{t_{1}}\|\widehat{X}(s)\|_{2}^{p} d s\right] .
\end{aligned}
$$

Substituting this into (G.7) produces

$$
\begin{aligned}
& \mathbb{E}\left[\sup _{0 \leq t \leq t_{1}}\|\widehat{c}(t)-c(t)\|_{2}^{p}\right] \\
& \leq \xi\left\{\mathbb{E}\|\widehat{c}(0)-c(0)\|_{2}^{p}+\mathbb{E}\left[\int_{0}^{t_{1}}\left\|\nabla_{s} c(s)\right\|_{2} d s\right]^{p}\right. \\
& \left.\quad+\int_{0}^{t_{1}} \mathbb{E}\|\widehat{X}(s)\|_{2}^{p} d s+\mathbb{E}\left[\int_{0}^{t_{1}}\|\widehat{X}(s)\|_{2} d s\right]^{p}\right\} .
\end{aligned}
$$

Using the well-known Hölder inequality again reveals

$$
\begin{aligned}
& \mathbb{E}\left[\sup _{0 \leq t \leq T}\|\widehat{c}(t)-c(t)\|_{2}^{p}\right] \\
& \leq \xi\left\{\mathbb{E}\|\widehat{c}(0)-c(0)\|_{2}^{p}+\int_{0}^{T} \mathbb{E}\|\widehat{X}(t)\|_{2}^{p} d t+\int_{0}^{T} \mathbb{E}\left\|\nabla_{t} c(t)\right\|_{2}^{p} d t\right\} .
\end{aligned}
$$

Thus, by Assumptions 21 and 22, we have, for $\forall \varepsilon>0$,

$$
\mathbb{E}\|\widehat{c}(0)-c(0)\|_{2}^{p} \leq \frac{\varepsilon}{3 \xi}, \quad \int_{0}^{T} \mathbb{E}\left\|\nabla_{t} c(t)\right\|_{2}^{p} d t \leq \frac{\varepsilon}{3 \xi} .
$$

Similar to Theorem 14, if there exists $\delta(T)>0$ such that when $\vee_{i=1}^{I} \hat{x}^{i}<\delta(T)$, we must have

$$
\int_{0}^{T} \mathbb{E}\|\widehat{X}(t)\|_{2}^{p} d t \leq \frac{\varepsilon}{3 \xi}
$$

Thus, to sum up

$$
\mathbb{E}\left[\sup _{0 \leq t \leq T}\|\widehat{c}(t)-c(t)\|_{2}^{p}\right] \leq \frac{\varepsilon}{3}+\frac{\varepsilon}{3}+\frac{\varepsilon}{3}=\varepsilon .
$$

Moreover, if we have $\lim _{T \rightarrow \infty} \delta(T)=0$, then we get

$$
\lim _{T \rightarrow \infty} \mathbb{E}\left[\sup _{0 \leq t \leq T}\|\widehat{c}(t)-c(t)\|_{2}^{p}\right]=0,
$$


which yields by using the well-known Levi lemma

$$
\mathbb{E}\left[\lim _{T \rightarrow \infty} \sup _{0 \leq t \leq T}\|\widehat{c}(t)-c(t)\|_{2}^{p}\right]=0,
$$

which gives the desired result.

\section{Conflict of Interests}

The author declares that there is no conflict of interests regarding the publication of this paper.

\section{Acknowledgment}

The author is very grateful for helpful comments and suggestions, which have greatly improved the quality of the paper, from the anonymous referee. Any remaining errors are, of course, my own responsibility.

\section{References}

[1] J. Wang, "The term structure of interest rates in a pure exchange economy with heterogeneous investors," The Journal of Financial Economics, vol. 41, no. 1, pp. 75-110, 1996.

[2] H. Yan, "Natural selection in financial markets: does it work?" Management Science, vol. 54, no. 11, pp. 1935-1950, 2008.

[3] H. Chen, S. Joslin, and N.-K. Tran, "Rare disasters and risk sharing with heterogeneous beliefs," NBER Working Paper no. 16035, 2010.

[4] T. Li, "Heterogeneous beliefs, asset prices, and volatility in a pure exchange economy," Journal of Economic Dynamics \& Control, vol. 31, no. 5, pp. 1697-1727, 2007.

[5] S. Basak and B. Croitoru, "Equilibrium mispricing in a capital market with portfolio constraints," Review of Financial Studies, vol. 13, no. 3, pp. 715-748, 2000.

[6] G. J. Mailath and A. Sandroni, "Market selection and asymmetric information," Review of Economic Studies, vol. 70, no. 2, pp. 343-368, 2003.

[7] L. Blume and D. Easley, "If you're so smart, why aren't you rich? Belief selection in complete and incomplete markets," Econometrica, vol. 74, no. 4, pp. 929-966, 2006.

[8] L. Kogan, S. A. Ross, J. Wang, and M. M. Westerfield, “The price impact and survival of irrational traders," Journal of Finance, vol. 61, no. 1, pp. 195-229, 2006.

[9] J. Mossin, "Optimal multiperiod portfolio policies," Journal of Business, vol. 3, pp. 373-413, 1968.

[10] N. H. Hakansson, "Convergence to isoelastic utility and policy in multiperiod portfolio choice," Journal of Financial Economics, vol. 1, no. 3, pp. 201-224, 1974.

[11] S. A. Ross, "Portfolio turnpike theorems for constant policies," Journal of Financial Economics, vol. 1, no. 2, pp. 171-198, 1974.

[12] G. Huberman and S. A. Ross, "Portfolio turnpike theorems, risk aversion, and regularly varying utility functions," Econometrica, vol. 51, no. 5, pp. 1345-1361, 1983.

[13] X. Jin, "Consumption and portfolio turnpike theorems in a continuous-time finance model," Journal of Economic Dynamics \& Control, vol. 22, no. 7, pp. 1001-1026, 1998.

[14] P. H. Dybvig, L. C. G. Rogers, and K. Back, "Portfolio turnpikes," Review of Financial Studies, vol. 12, no. 1, pp. 165-195, 1999.
[15] C. Huang and T. Zariphopoulou, “Turnpike behavior of longterm investments," Finance and Stochastics, vol. 3, no. 1, pp. 1534, 1999.

[16] D. G. Champernowne, "Some implications of golden age conditions when savings equal profits," Review of Economic Studies, vol. 29, no. 3, pp. 235-237, 1962.

[17] I. F. Pearce, "The end of the golden age in Solovia: a further fable for Growthmen hoping to be "one up" on Oiko," The American Economic Review, vol. 52, pp. 1088-1097, 1962.

[18] E. S. Phelps, "The golden rule of accumulation: a fable for growthmen," American Economic Review, vol. 51, pp. 638-643, 1961.

[19] E. S. Phelps, "The end of the golden age in Solovia: comment," American Economic Review, vol. 52, pp. 1097-1099, 1962.

[20] E. S. Phelps, "Second essay on the golden rule of accumulation," American Economic Review, vol. 55, pp. 793-814, 1965.

[21] P. A. Samuelson, "A Catenary Turnpike theorem involving consumption and the golden rule," American Economic Review, vol. 55, pp. 486-496, 1965.

[22] R. C. Merton, "An intertemporal capital asset pricing model," Econometrica, vol. 41, pp. 867-887, 1973.

[23] J. Lucas Jr., "Asset prices in an exchange economy," Econometrica, vol. 46, no. 6, pp. 1429-1445, 1978.

[24] J. C. Cox, J. E. Ingersoll Jr., and S. A. Ross, "An intertemporal general equilibrium model of asset prices," Econometrica, vol. 53, no. 2, pp. 363-384, 1985.

[25] L. G. Epstein and T. Wang, "Intertemporal asset pricing under knightian uncertainty," Econometrica, vol. 62, pp. 283-322, 1994.

[26] D. Duffie and W. Zame, "The consumption-based capital asset pricing model," Econometrica, vol. 57, no. 6, pp. 1279-1297, 1989.

[27] F. Kubler and K. Schmedders, "Stationary equilibria in assetpricing models with incomplete markets and collateral," Econometrica, vol. 71, no. 6, pp. 1767-1793, 2003.

[28] L. Blume and D. Easley, "Evolution and market behavior," Journal of Economic Theory, vol. 58, no. 1, pp. 9-40, 1992.

[29] G. Y. Luo, "Market efficiency and natural selection in a commodity futures market," Review of Financial Studies, vol. 11, pp. 647-674, 1998.

[30] A. Sandroni, "Do markets favor agents able to make accurate predictions?" Econometrica, vol. 68, no. 6, pp. 1303-1341, 2000.

[31] C. Chiarella and X. He, "Asset price and wealth dynamics under heterogeneous expectations," Quantitative Finance, vol. 1, no. 5, pp. 509-526, 2001.

[32] C. Chiarella, R. Dieci, and L. Gardini, "Asset price and wealth dynamics in a financial market with heterogeneous agents," Journal of Economic Dynamics \& Control, vol. 30, no. 9-10, pp. 1755-1786, 2006.

[33] M. Anufriev, "Wealth-driven competition in a speculative financial market: examples with maximizing agents," Quantitative Finance, vol. 8, no. 4, pp. 363-380, 2008.

[34] M. Anufriev and P. Dindo, "Wealth-driven selection in a financial market with heterogeneous agents," Journal of Economic Behavior and Organization, vol. 73, no. 3, pp. 327-358, 2010.

[35] S. Brianzoni, C. Mammana, and E. Michetti, "Wealth distribution in an asset pricing model: the role of the switching mechanism," Applied Mathematical Sciences, vol. 6, no. 9-12, pp. 423-442, 2012.

[36] T. Bewley, "An integration of equilibrium theory and turnpike theory," Journal of Mathematical Economics, vol. 10, no. 2-3, pp. 233-267, 1982. 
[37] M. Yano, "The turnpike of dynamic general equilibrium paths and its insensitivity to initial conditions," Journal of Mathematical Economics, vol. 13, no. 3, pp. 235-254, 1984.

[38] M. Yano, "Stability and turnpike theorems in dynamic competitive equilibrium," Japanese Economic Review, vol. 50, no. 4, pp. 398-421, 1999.

[39] M. Yano, "On the dual stability of a von Neumann facet and the inefficacy of temporary fiscal policy," Econometrica, vol. 66, no. 2, pp. 427-451, 1998.

[40] A. Kondo, "On the inefficacy of temporary policy in a dynamic general equilibrium with money," The Japanese Economic Review, vol. 59, no. 3, pp. 324-344, 2008.

[41] L. W. McKenzie, "Turnpike theory, discounted utility, and the von Neumann facet," Journal of Economic Theory, vol. 30, no. 2, pp. 330-352, 1983.

[42] J. A. Scheinkman, "On optimal steady states of $n$-sector growth models when utility is discounted," Journal of Economic Theory, vol. 12, no. 1, pp. 11-30, 1976.

[43] D. Dai, "Stochastic versions of turnpike theorems in the sense of uniform topology," Annals of Economics and Finance, vol. 13, no. 2, pp. 389-431, 2012.

[44] M. Yano, "Competitive equilibria on turnpikes in a McKenzie economy. I. A neighborhood turnpike theorem," International Economic Review, vol. 25, no. 3, pp. 695-717, 1984.

[45] M. Yano, "Competitive equilibria on turnpikes in a McKenzie economy. II. An asymptotic turnpike theorem," International Economic Review, vol. 26, no. 3, pp. 661-669, 1985.

[46] I. Karatzas and S. E. Shreve, Brownian Motion and Stochastic Calculus, vol. 113 of Graduate Texts in Mathematics, Springer, New York, NY, USA, 2nd edition, 1991.

[47] D. J. Higham, X. Mao, and A. M. Stuart, "Strong convergence of Euler-type methods for nonlinear stochastic differential equations," SIAM Journal on Numerical Analysis, vol. 40, no. 3, pp. 1041-1063, 2002. 


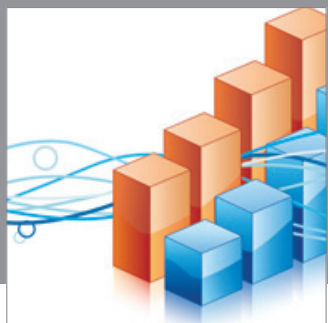

Advances in

Operations Research

mansans

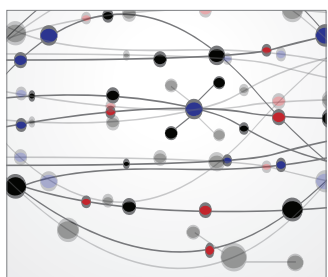

The Scientific World Journal
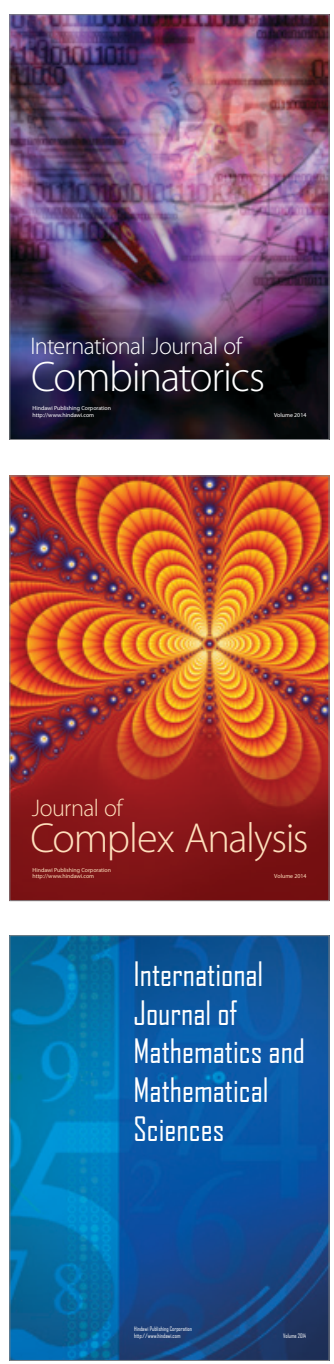
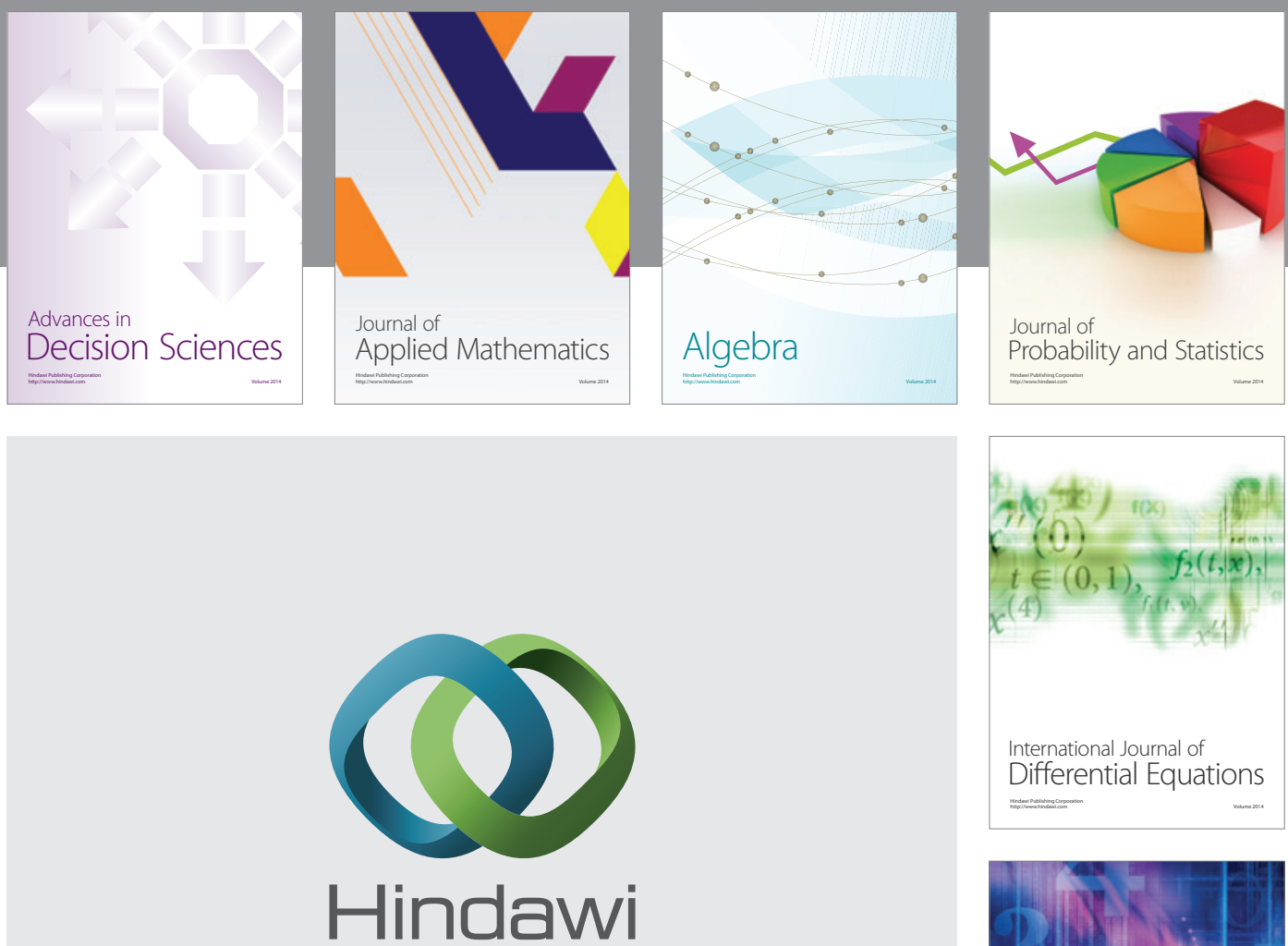

Submit your manuscripts at http://www.hindawi.com
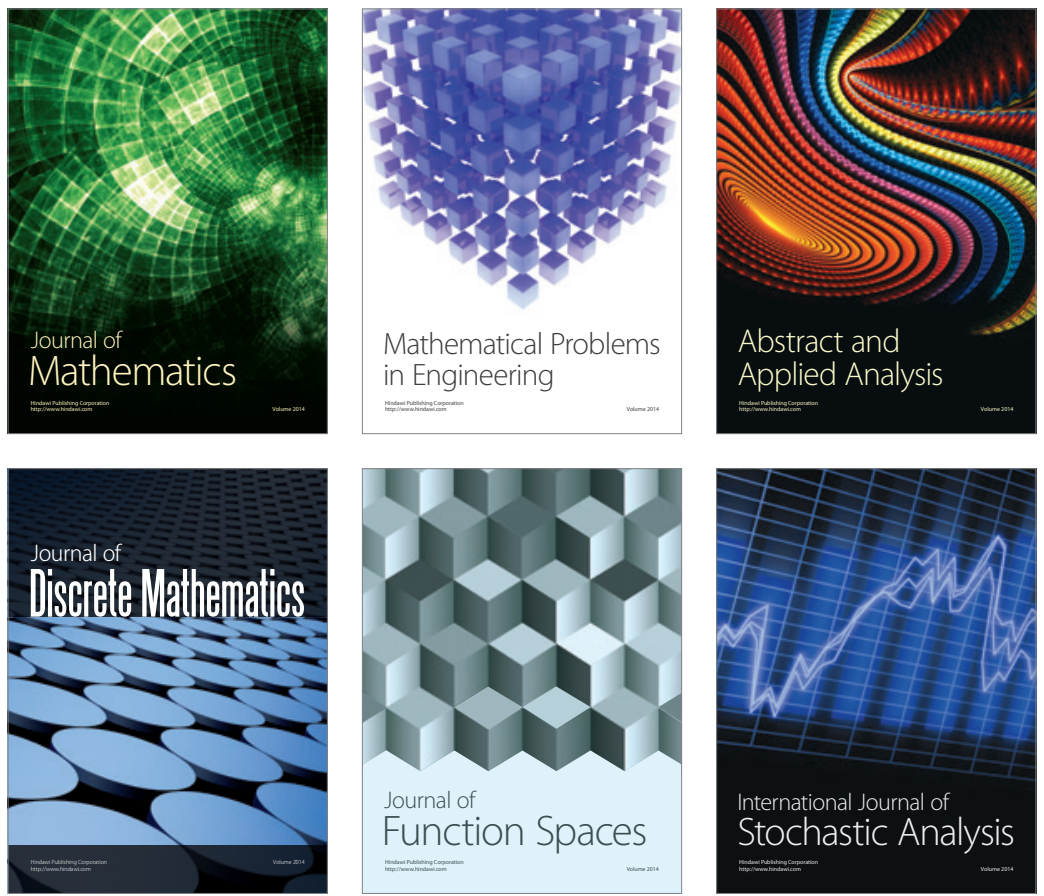

Journal of

Function Spaces

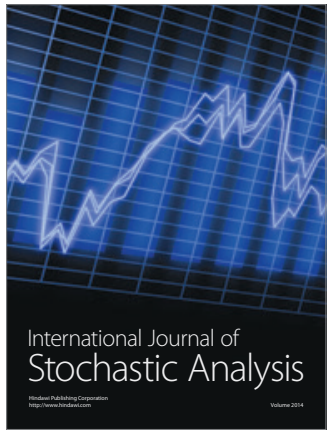

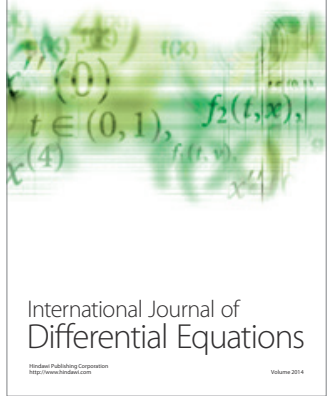
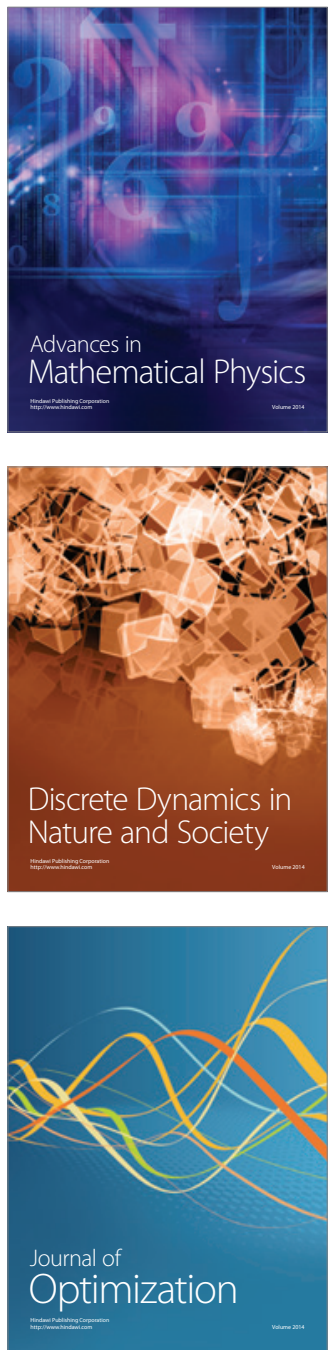